\title{
The APEX Method and Real-Time Blind Deconvolution of Scanning Electron Microscope Imagery
}

\author{
Alfred S. Carasso* \\ David S. Bright ${ }^{\dagger}$ \\ András E. Vladár ${ }^{\ddagger}$ \\ U.S. DEPARTMENT OF COMMERCE \\ Technology Administration \\ Mathematical and Computational Sciences Division \\ Surface and Microanalysis Science Division \\ Precision Engineering Division \\ National Institute of Standards \\ and Technology \\ Gaithersburg, MD 20899
}

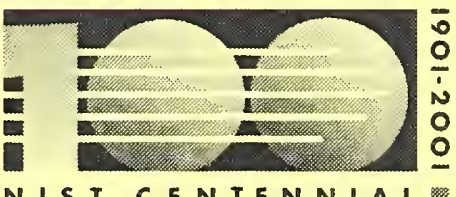





\title{
The APEX Method and Real-Time Blind Deconvolution of Scanning Electron Microscope Imagery
}

\author{
Alfred S. Carasso \\ David S. Bright ${ }^{\dagger}$ \\ András E. Vladár \\ U.S. DEPARTMENT OF COMMERCE \\ Technology Administration \\ Mathematical and Computational Sciences Division \\ Surface and Microanalysis Science Division \\ Precision Engineering Division \\ National Institute of Standards \\ and Technology \\ Gaithersburg, MD 20899
}

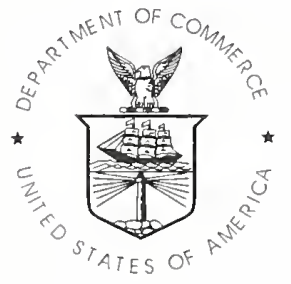

U.S. DEPARTMENT OF COMMERCE

Donald L. Evans, Secretary

TECHNOLOGY ADMINISTRATION

Phıllıp J. Bond, Under Secretary

of Commerce for Technology

NATIONAL INSTITUTE OF STANDARDS

AND TECHNOLOGY

Karen $\mathrm{H}$. Brown, Acting Director 



\title{
The APEX method and real-time blind deconvolution of scanning electron microscope imagery
}

\author{
Alfred S. Carasso* David S. Bright ${ }^{\dagger} \quad$ András E. Vladár ${ }^{\ddagger}$
}

\begin{abstract}
Loss of resolution due to image blurring is a major concern in electron microscopy. The point spread function describing that blur is generally unknown. This paper discusses the use of a recently developed FFT-based direct (non-iterative) blind deconvolution procedure, the APEX method, that can process $512 \times 512$ images in less than a minute on current (lesktop) platforms. The method is predicated on a restricted but significant class of shift-invariant blurs, consisting of finite convolution products of Lévy probability density functions. Such bhurs considerably generalize Gaussian and Lorentzian point sprear functions. In this paper, the method is applied to a variety of original SEM micrographs, and slown to be useful in enlancing and detecting fine detail not otherwise discernible. Quantitative sharpness analysis of 'ideal sample' micrographs, shows that APEX processing can actually produce sharper imagery than is achievable with optimal microscope settings.
\end{abstract}

Subject terms: electron microscopy, real-time, image deblurring, blind deconvohtion, Lévy density functions, APEX method, SECB method, SEM images.

\section{Introduction}

Loss of resolution due to image blurring is a major concern in scanning electron microscopy (SEM). Moreover, unless specifically measured, ${ }^{1,2}$ the shape of the electron beam is not known to the microscopist. Hence, the point spread function (psf) describing the blur is generally unknown. This paper discusses the use in electron microscopy of a recently developed blind deconvolution procedure, the APEX method,, 3 t which sharpens the image while simultaneously increasing contrast and brightness. The degree of enhancement can be adjusted by appropriate choice of imput parameters. To the extent permitted by the level of data noise, the APEX method sharpens the innage

${ }^{*}$ Mathematical and Computational Sciences Division, National Institute of Standards and Technology, Gaitlersburg, MD 20899. (alfred.carasso(inist.gov)

†'Surface and Microanalysis Science Division, National Institute of Standards and Technology, Gaithershurg, MD 20899. (david.bright@inist.gov)

${ }^{\ddagger}$ Precision Engineering Division, National Institute of Standards and Teclnology, Gaithersburg, MD 20899. (andras.vladar(unist.gor) 
by restoring some of the high frequency content that had been attenuated in the comse of inaging the sample. In this paper, the method is applied to a variety of original SEM micrographs and shown to be useful in enhancing and detecting fine detail not otherwise discernible. In addition. quantitative sharpness analysis of 'ideal sample' micrographs, ${ }^{2}$ shows that APEX processing can actually produce sharper imagery than is achievable with optimal inicroscope settings.

As in all inverse problems, successful use of the APEX method requires a-priori knowledge about the solution. Here, such prior knowledge takes the form of training and experience on the part of the microscopist, whose judgment is called upon to distinguish genuine features in the presence of noise and visually select the 'optimal' reconstruction. The images we are concerned with come from scanning electron beam instruments sucle as the field emission gun scamming electron microscope (FEGSEM), a high resolution instrument, and the environmental scanning electron microscope (ESEM), a lower resolution instrument with more flexible sample handling capability. In a future report, we shall explore the possible use of APEX methodology to produce a quantitative measure of SEM inlaging performance.

Blind deconvolution seeks to deblur an image without knowing the cause of the blur. This is a difficult mathematical problem in which ill-conditioning is compounded with non-uniqueness of solutions. A priori constraints reduce, but do not entirely eliminate, the multiplicity of solutions. While many of these solutions are physically meaningless, there are in general several useful solutions. ${ }^{t}$ Most approaches to blind deconvolution are iterative in nature, and aim at simultaneous recoustruction of both the psf and the deblurred image. However, that iterative process may become ill-beliaved and develop stagnation points or diverge altogether. ${ }^{3}$ When the iterative process is stable, several thousand iterations may be necessary to resolve fine detail. In general, iterative algorithms are not well-suited for real-time processing of large size images of complex objects.

The APEX nothod is an FFT-based direct (non-iterative) blind deconvolution technique that can be used in real-time applications. It was developed and analyzed in Ref. 3, and documented there with mumerous applications to synthetically blurred images. More recently, ${ }^{4}$ the method was successfully applied to a variety of real blurred images obtained from diverse imaging modalities, including astrononical, acrial, and Landsat inages, MRI and PET brain scans, as well as other types of interesting images. However, not all images can be usefully enhanced witlo the APEX method.

Rather than considering the blind deconvolution problem in full generality. the APEX method is predicated on a restricted but significant class of shift-invariant blurs, the class $\mathbf{G},{ }^{5-7}$ which consists of finite convolution products of 2-D radially synmetric Lévy 'stable' probability density functions. That class considerably generalizes Gaussian and Lorentzian psf's. The motivation for using the class $\mathrm{G}$ as the framework for the APEX method, is that numerous electron-optical imaging devices liave psfs in class $G$. or have psfs that can be well-approximated by class $G$ psfs. This is documented in section 2. Apparently, as will be slown in sections 7 and 8 below, the class $\mathbf{G}$ can also be usefully applied to electron microscope imagery.

The APEX method is based on detecting the signature of a class G psf from 1-D Fourier analysis of the blurred image. That detected psf is then used in a separate FFT-based lirect image deblurring procedure, the SECB method, ${ }^{5}$ to produce the deblurred image.* When the APEX method is useful, blind deconvolution of $512 \times 512$ images can be acconplished in less than a nimute

\footnotetext{
${ }^{*}$ United States patents have been issued on parts of the work described in Refs. $3-7$.
} 
A

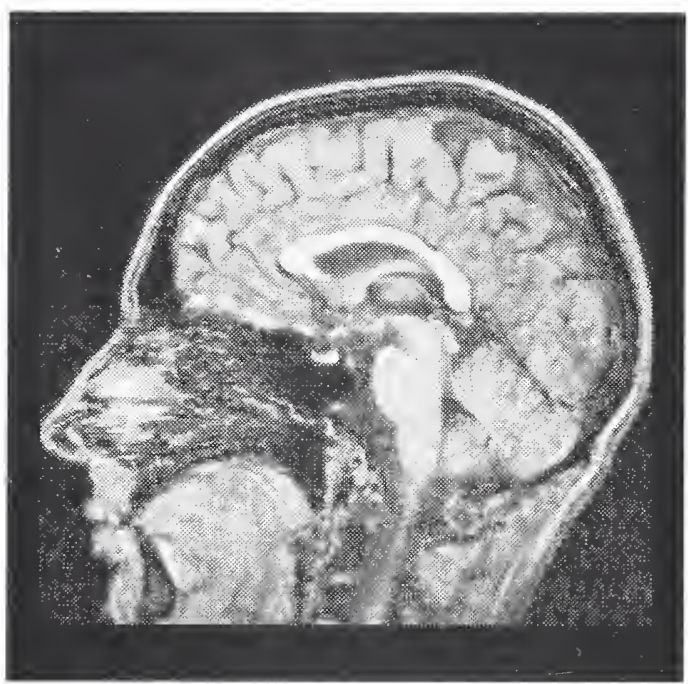

C

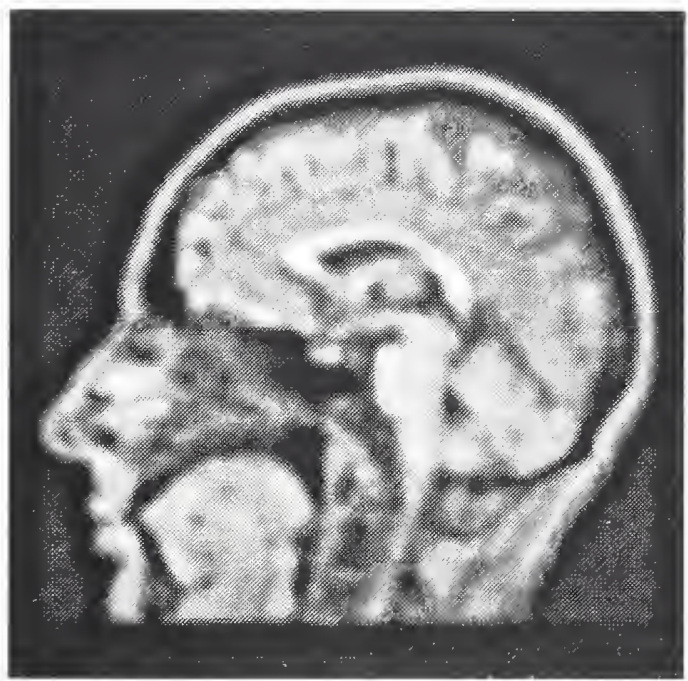

B

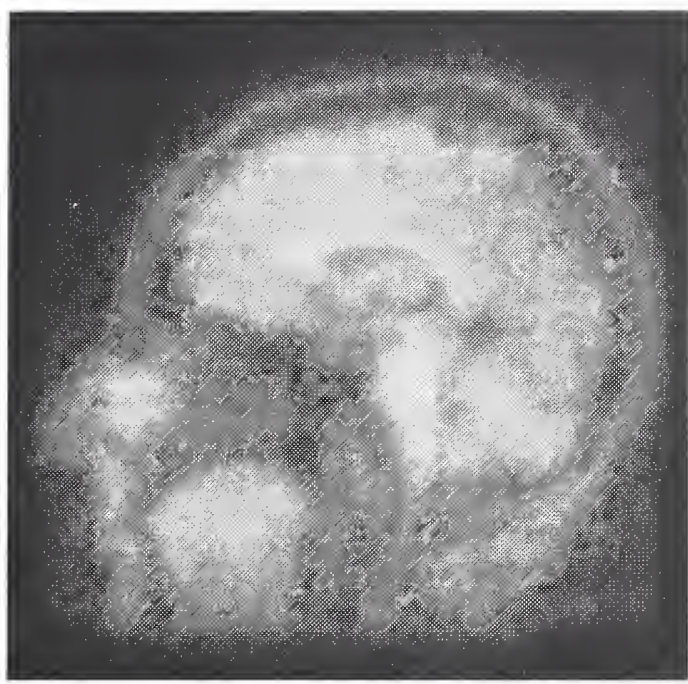

D



Figure 1: Comparing APEX processing with unsharp masking on a synthetically bhurred inage. (A) Original 8-bit $512 \times 512$ MRI sagittal brain image. (B) Synthetically blurred MRI inage stored in 8-bit precision. (C) Sharpening of image (B) using unsharp masking. (D) Sharpening of image (B) using APEI method. 
on current desktop platforms. As illustrated in Figure 1, APEX processing can produce significantly sharper images than is generally possible with unsharp masking.

An important aspect of blind deconvolution can be illustrated by means of the following analogy. Inagine several experiencet photographers located at approximately the same vantage point, and simultaneously photographing an identical scene. In general, different images will be produced through use of different cameras, film, light filters, exposures, printing, and the like. While each image is a correct visual representation of the original scene, the images will differ from one another in contrast, brightness, sharpness, and numerous other details. A pixel by pixel comparison of these images would reveal substantial differences. Which of the several photographs is the true version of reality cannot easily be answered. They are all useful approximations. An analogous phenomenon occurs in blind deconvolution. As illustrated in section 4, given a blurred image, there are in general many useful reconstructions that are possible. These reconstructions nay differ substantially from one another at individual pixels, while being correct visual representations of the object that was imaged. This is an inherent non-uniqueness property of the blind deconvolution problem, independently of any particular algorithm that might be used to solve that problem. ${ }^{4}$

A basie property of the APEX method is that it generally provides several psfs that can be used to obtain useful reconstructions of the same blured image. As in the case above, these reconstructions differ from ore another at individual pixels while being visually correct. As already noted, a-priori knowledge about the desired solution is a necessary ingredient for solving ill-posed inverse problems. Such knowledge is expected to guide the user in his selection of the best reconstruction. Whether or not APEX processing is beneficial in any given case can nsually be quickly decided. For innages where APEX processing provides useful enhancement, fine tuning of parameters enables the user to adjust the quality of the reconstruction, within the limitations imposed by the level of noise in the blurred image.

\section{Imaging systems, Lévy point spread functions, and the class G}

Point spread functions $h(x, y)$ can be viewed as 2-D probability density functions since they are nonnegative and integrate to mnity. The Fourier transform $h(\xi, \eta)$ of a psf $h(x, y)$ is called the optical transfer function (otf). Knowledge of the otf determines the psf and vice versa. Note that while the psf $h(x, y)$ is always non-negative, the otf $\hat{h}(\xi, \eta)$ is complex-valued in gereral. The absolute value of the otf is called the modulation transfer function (mtf).

Gaussian psfs are ubiquitous in imaging systems but represent only one example of the general class of Lévy stable densities. In the 2-D radially symmetric case, Lévy stable densities $h(x, y)$ can be clefined implicitly in terms of their Fourier transforms by

$$
\hat{h}(\xi, \eta) \equiv \int_{R^{2}} h(x, y) e^{-2 \pi i(\xi, x+\eta y)} d x d y=e^{-\alpha\left(\xi^{2}+\eta^{2}\right)^{\beta}}, \quad \alpha>0, \quad 0<\beta \leq 1 .
$$

For general $\beta, h(x, y)$ in $(1)$ is not known in closed form. However, the cases $\beta=1$ and $\beta=1 / 2$ correspond to Ganssian and Lorentzian (or Canchy) densities respectively. When $\beta=1, h(x, y)$ lias stim tails and finite variance. For $0<\beta<1, h(x, y)$ has fat tails and infinite variance. The occurence and analysis of Lévy processes in the physical sciences are subjects of significant current interest. ${ }^{9-12}$ 
Image intensifiers, CCDs, and mumerous other electron-optical devices are used in a wide variety of astrononical, industrial, biomedical, military, and surveillance imaging systems. A systematic study of electron-optic mif measurements has led to the important empirical discovery, ${ }^{13-15}$ that an extensive variety of electronic inaging devices have otfs $h(\xi, \eta)$ that are well-described by (1) with $1 / 2 \leq \beta \leq 1$. In particular, non-Gaussian behavior is often found in electron-optic inlaging systems. For any given device. the values of a and $\beta$ can be determined using specialized graph paper. The characterization (1) is useful in other areas of optics. The diffraction-limited otf for a perfect lens $[16$, p. 154], can be approxinnated over a wide frequency range by (1), with $\beta=3 / 4$ and $\alpha$ a function of the cutoff frequency. ${ }^{17}$ The otf for long-exposure inaging through atmospheric turbulence, ${ }^{18}$ is known to be given by $(1)$ with $\beta=5 / 6$, and $\alpha$ deternined by atnospheric conditions. In Ref. 19, ntf data for 56 different kinds of photographic film are analyzed. Good agreenent is found when these data are fitted with (1), and the pairs $(\alpha, \beta)$ characterizing each of these $56 \mathrm{mt}$ fs are identified. It is found that 36 types of film have mifs where $1 / 2 \leq \beta \leq 1$. The remaining 20 types have mtfs with values of $\beta$ in the range $0.265 \leq \beta \leq 0.475$.

For cascaded imaging systems composed of several elements satisfying (1), the resulting lumperl otf has the form

$$
\hat{h}(\xi, \eta)=e^{-\sum_{i=1}^{J} \alpha_{l}\left(\xi^{2}+\eta^{2}\right)^{\beta_{i}}}, \quad \alpha_{i} \geq 0, \quad 0<\beta_{i} \leq 1 .
$$

Such an expression can also be used to best-fit a large variety of empirically obtained optical transfer functions, by varying the parameters $\alpha_{i}, \beta_{i}$, and $J$. We define $\mathrm{G}$ to be the class of all point spread functions $h(x, y)$ satisfring $(2)$. Note that class $\mathrm{G}$ psfs have non-negative Fouricr transforms. This is not true of psfs in general. For example, the optical transfer function for uniform optical defocus blur is the 'sombrero function' [20, p. 72], which develops negative oscillations.

Motivated by these considerations, we consider inage deblurring problems with psfs in G. In the absence of noise, we liave

$$
H f_{e} \equiv \int_{R^{2}} h(x-u, y-v) f_{e}(u, v) d u d v \equiv h(x, y) \otimes f_{e}(x, y)=g_{e}(x, y),
$$

where $g_{e}(x, y)$ is the blurred image that would have been recorded in the absence of noise, $f_{e}(x, y)$ is the exact unblurred image, $h(x, y)$ is a point spread function in class $\mathrm{G}$, and $\otimes$ denotes convolution. In general, the given blurred image $g(x, y)$ includes noise, which is viewed as a separate additional degradation,

$$
g(x, y)=g_{e}(x, y)+n(x, y) .
$$

Here. $n(x, y)$ represents the cumulative effects of all errors affecting final acquisition of the digitized array $g(x, y)$. This includes multiplicative noise. where $n(x, y)$ may be a nonlinear function of $f_{e}(x, y)$. Neither $g_{e}(x, y)$ nor $n(x, y)$ are actually known, only their sum $g(x, y)$. Hence, rather than (3), we must consider the more difficult problem

$$
H f \equiv h(x, y) \otimes f(x, y)=g(x, y) .
$$

As is well-known, ${ }^{22}$ even though $n(x, y)$ nllay be presumed small, its presence in (4) has a profound impact on the solution of the ill-posed equation (5). A survey of the best-known lincar and nonlinear algorithms for handling (5) may be found in Ref. 7 . The strategy is to find an approxinlate solution $f^{\dagger}(x, y)$ such that $h(x, y) \otimes f^{\dagger}(x, y) \approx g(x, y)$ and such that $\left\|f^{\dagger}-f_{e}\right\|$ is small. For psfs in class $\mathrm{G}$, the SECB method outlined in section 3 is particularly effective. 


\subsection{Connection with SEM imaging}

In interpreting (3) in relation to SEM imaging, a conceptual framework that has been used in several recent studies ${ }^{23}$ is helpful. Let $s(x, y)$ be a function describing the actual sample. The SEM converts $s(x, y)$ into an image $i(x, y)$, where

$$
i(x, y)=I[s(x, y)]
$$

Here, $I$ is the instrument transform and is partly nonlinear. The nonlinear component of $I$, call it $M$, consists of the details of the nonlinear interaction between the electrons and the material. This component can be studied ${ }^{23,24}$ by Monte Carlo simulations applied to electron trajectories, but is not readily invertible. The other component of $I$, call it q, describes blurring due to the electron beam point spread, along with sone of the instrument's electronics. That component is often represented as a convolution. Therefore, in the absence of noise,

$$
i(x, y)=q(x, y) \otimes M[s(x, y)]
$$

Comparing (7) with (3), we are led to identify $i(x, y)$ with $g_{e}(x, y), M[s(x, y)]$ with $f_{e}(x, y)$, and $q(x, y)$ with $h(x, y)$. Thus, blind deconvolution of (5) using the APEX method, may be interpreted as an attempt to recapture $M[s(x, y)]$ from noisy data, by undoing blurring due primarily to the unknown electron beam point spread.

\section{Deblurring with the SECB method}

The SECB method is a direct FFT-based image deblurring technique designed for equations of the form (5) when $h(x, y)$ is known and belongs to G. A complete discussion of that method, together with error bounds and comparisons with other methods, may be found in Refs. 5-7. Significantly, the SECB method does not impose smoothness requirements, such as a-priori bounds on the Laplacian or other derivatives of the unknown image $f(x, y)$. This is an important consideration since many intages have sharp edges and other localized non-differentiable features.

For class G psfs, we may define fractional powers $H^{t}, 0 \leq t \leq 1$, of the convolution integral operator $H$ in $(5)$ as follows

$$
H^{t} f \equiv \mathcal{F}^{-1}\left\{h^{\dagger}(\xi, \eta) \hat{f}(\xi, \eta)\right\}, \quad 0 \leq t \leq 1
$$

Class G psfs are intimately related to diffusion processes, and solving (5) is equivalent to finding the initial value $u(x, y, 0)=f(x, y)$ in the backwards in time problem for the generalized diffusion equation

$$
\begin{aligned}
& u_{i}=-\sum_{i=1}^{J} \lambda_{i}(-\Delta)^{\beta_{i}} u, \quad \lambda_{i}=\alpha_{i}\left(4 \pi^{2}\right)^{-\beta_{i}}, \quad 0<t \leq 1 . \\
& u(x, y, 1)=g(x, y) .
\end{aligned}
$$

When this initial value $f(x, y)$ is known, $u(x, y, t)=H^{t} f$ is the solution of $(9)$ at time $t$. The SECB method is a regularization method for solving the ill-posed problem (9) that takes into account the presence of noise in the blurred image data $g(x, y)$ at $t=1$. The SECB deblurred innage $f^{\dagger}(x, y)$ is 
an approximation to $f_{c}(x, y)$ that is obtained in closed form in Fourier space. With $\bar{z}$ denoting the complex conjugate of $\approx$,

$$
f^{\dagger}(\xi, \eta)=\frac{\overline{\hat{h}}(\xi, \eta) \hat{g}(\xi, \eta)}{|\hat{h}(\xi, \eta)|^{2}+K^{r-2}\left|1-\hat{h}^{s}(\xi \cdot \eta)\right|^{2}},
$$

leading to $f^{\dagger}(x, y)$ upon inverse transforming. Here, the regularization parameters $K, s$ are positive constants that are chosen based on a-priori information. ${ }^{5-7}$ In blind deconvolution applications of the SECB method, the APEX-detected paraneters $\alpha_{i}$, $\beta_{i}$ are used in (2) which is then input into (10). In practice, FFT algorithms are used to obtain $f^{\dagger}(x, y)$. This may result in indivirlual pixel values that are negative. Accordingly, all negative values are reset to the value zero. For $512 \times 512$ images, a single trial SECB restoration requires about one second of cpu time on current (leskitop) workstations. We may also form and display

$$
t^{\dagger}(x, y, t)=H^{t} f^{\dagger}(x, y),
$$

for selected decreasing values of $t$ lying between 1 and 0 . This simulates marching backwards in time in (9), and allows monitoring the gradual deblurring of the image. As $t \rightarrow 0$ the partial restorations $u^{\dagger}(x, y, t)$ become sharper. However. noise and other artifacts typically become more noticeable as $t \rightarrow 0$. Such slow motion deconvolution allows detcetion of features in the inage before they become obscured by noise or ringing artifacts. As will be seen below, marching backwards in time is an important element in the APEX method.

\section{Non-uniqueness in blind deconvolution}

Blind deconvolution of inages is a mathematical problem that is not fully understoot. Welldocumented examples of the kinds of behavior that may occur are of particular interest. In this section, we highlight important non-uniqueness aspects of that problem that are lielpful in understanding the results of the APEX method. Let $f_{e}(x, y)$ be a given exact sharp image, let $h(x, y)$ be a Lévy point spread function, and let $g(x, y)=h(x, y) \otimes f_{e}(x, y)+$ noise. We sliall show that given the blurred image $g(x, y)$, there are in general many point spread functions $h,(x, y) \neq h(x, y)$ that deblur $g(x, y)$, producing useful reconstructions $f_{i}(x, y) \neq f_{e}(x, y)$, with $h_{i}(x, y) \otimes f_{i}(x, y) \approx g(x, y)$.

The sharp $512 \times 512$ New Orleans cathedral image $f_{e}(x, y)$ in Figure 2(A) was syntletically blurred by convolution with a Cauchy density $h(x, y)$ with $\alpha_{0}=0.075, \beta_{0}=0.5$. This produced the blurref image $g(x, y)$ in Figure 2(B). In this experiment, $g(x, y)$ was computed and stored in 16-bit precision. Thus, $g(x, y)$ differs from $g_{e}(x, y)$ by the effects of 16 -bit rounding noise. Doblurring that image with the correct psf values $\alpha=0.075, \beta=0.5$, produces Figure 2(C). As expected, this is in excellent visual agrecment with $f_{e}(x, y)$ in Figure 2(A). However, Figure 2(D) is another useful enhancenent of Figure $2(\mathrm{~B})$. It was obtained using a Lévy density with values $(\alpha, \beta)$ where $\alpha>\alpha_{0}, \beta<\beta_{0}$, and it differs from Figure 2(A) in contrast, brightness, and sharpmess of dletail. Here, $\alpha=0.239767, \beta=0.385568$. Note the indented 'blind window' highlighted in the left lateral tower in Figure 2(D). This architectural detail is barely discornible in Figure 2(A), and not identifiable in Figure 2(C). Both deblurred intages (C) and (D) were obtained using the SECB method with $s=0.001$ and $K=100$. One dimensional cross sections of the two rlistinct psfs used in Figure 2 are displayed in Figure 3. To facilitate comparison, the two psis in Figure 3 are nomalizer 
A



C

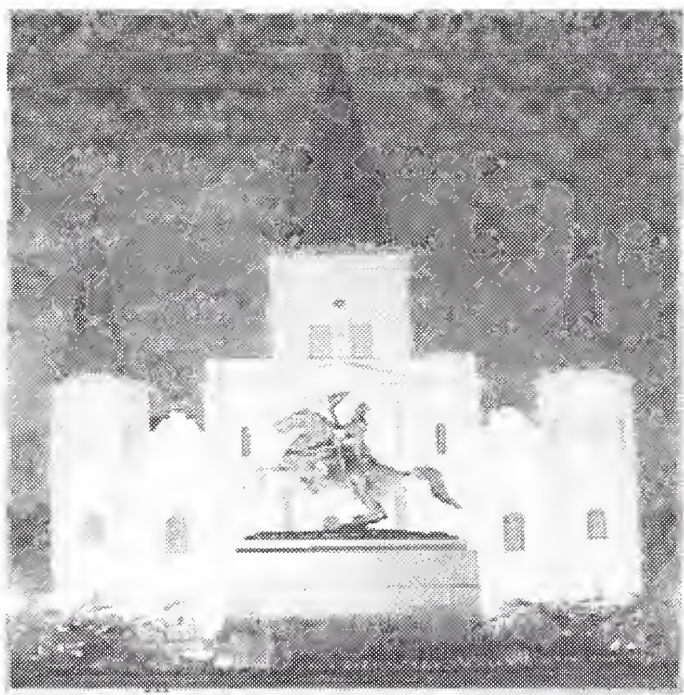

B



D

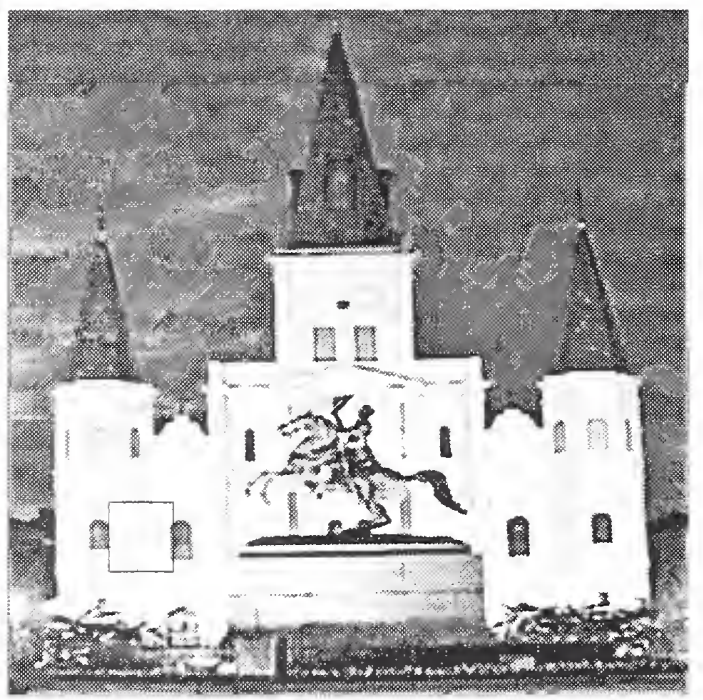

Figure 2: Non-miqueness in blind deconvolution. Distinct point spread functions exist that produce useful reconstructions from the same blurred intage. (A) Original slarp $512 \times 512$ New Orleans cathedral intage. (B) 16-bit synthetically blurred image created by convolution with Lorentzian density with $\alpha=0.075, \beta=0.5$. (C) Deblurring of image (B) using correct parameters $\alpha=$ $0.075, \beta=0.5$. (D) Deblurring of image (B) using $\alpha=0.239767, \beta=0.385568$. Image (D) differs from image (C) in contrast, brightness and sharpness of detail. In particular, indented 'blind window' highlighted in left lateral tower in image (D), is not discernible in image (C). Deblurred images obtained using SECB procedure with $s=0.001$ and $K=100$. 


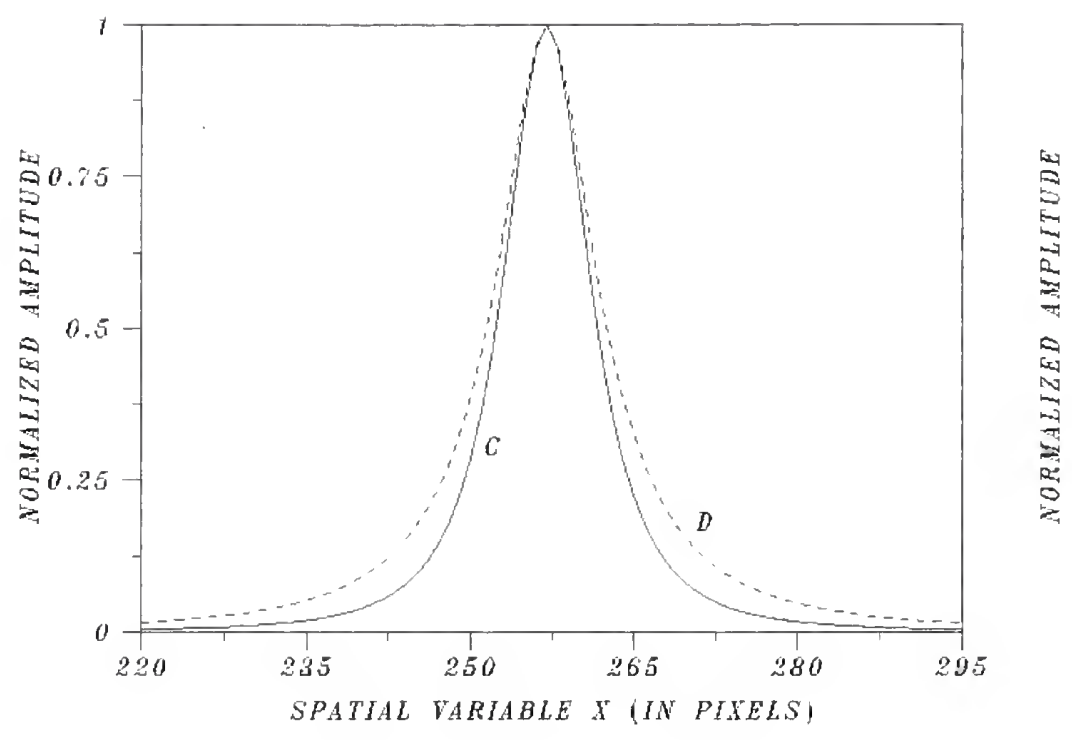

Figure 3: Two distinct point spread functions that deblur image (B) in Figure 1. Curves C and D are 1 -D cross sections of the $512 \times 512$ psfs that respectively produced images (C) and (D) in Figure 2. To facilitate comparison, curves were normalized to unit maximum. These psfs also exhibit distinct heavy tail behavior. 
so as to have a maximum vahue of 1.0. These psfs also exhibit distinct heavy tail behavior not shown in Figure 3. There are obviously many other distinct psfs lying between these two curves that produce useful reconstructions. Convolution of each reconstruction with its corresponding psf in Figure 3, reproduces the blured inage in Figure 2(B), to within a small error.

\section{SEM images and convex Fourier transforms}

The APEX method is a blind deconvolution technique based on detecting class G psf signatures by appropriate 1-D Fonrier anatysis of the blurred image $g(x, y)$. The detected psf parameters are then input into the SECB algorithm to deblur the image. The Fourier transform is the primary mathematical tool in each of these steps. Aceordingly, the qualitative behavior in Fonrier space of a large class of SEM images is of interest.

Let $f_{e}(x, y)$ be an exact sharp image as in (3). Since $f_{e}(x, y) \geq 0$

$$
\left|\hat{f}_{e}(\xi, \eta)\right| \leq \int_{R^{2}} f_{e}(x, y) d x d y=f_{e}(0,0)=\sigma>0 .
$$

Also, since $g_{e}(x, y)=h(x, y) \otimes f_{e}(x, y)$ and $h(x, y)$ is a probability density,

$$
g_{e}(0,0)=\int_{R^{2}} g_{e}(x, y) d x d y=\int_{R^{2}} f_{e}(x, y) d x d y=\hat{f}_{e}(0,0)=\sigma>0 .
$$

Using $\sigma$ as a normalizing constant, we may normalize Fourier transform quantities $\hat{q}(\xi, \eta)$ by dividing by $\sigma$. Let

$$
\hat{q}^{*}(\xi, \eta)=\hat{q}(\xi, \eta) / \sigma
$$

denote the normalized quantity. The function $\left|\hat{f}_{e}^{*}(\xi, \eta)\right|$ is highly oscillatory, and $0 \leq\left|\hat{f}_{e}^{*}\right| \leq 1$. Since $f_{e}(x, y)$ is real, its Fourier transform is conjugate symmetric. Therefore, the function $\left|\hat{f}_{e}^{*}(\xi, \eta)\right|$ is symmetric about the origin on any line through the origin in the $(\xi, \eta)$ plane. The same is true for the bhurred image data $\left|g^{*}(\xi, \eta)\right|$.

All bhurred images in sections 5 through 8 are of size $512 \times 512$ and quantized at 8-bits per pixel. For any blurred inage $g(x, y)$, the discrete Fourier transform is a $512 \times 512$ array of complex numbers, which we again denote by $g(\xi, \eta)$ for simplicity. The 'frecuencies' $\xi, \eta$ are now integers lying between -256 and 256 , and the zero frequency is at the center of the transform array. This ordering is achieved by pre-multiplying $g(x, y)$ by $(-1)^{x+y}$. We shall be interested in the values of such transforms along single lines through the origin. The discrete transforms $\left|\hat{g}^{*}(\xi, 0)\right|$, and $\left|g^{*}(0, \eta)\right|$ are immediately available. Inage rotation may be used to obtain discrete transforms along other directions. All 1-D Fonrier donain plots shown in this paper are taken along the axis $\eta=0$ in the $(\xi, \eta)$ plane. In these plots, the zero frequency is at the center of the horizontal axis, and the graplis are necessarily symmetric about the vertical line $\xi=0$. Examples of such plots are slown in Figure 4.

The class of SEM images $g(x, y)$ considered in the present paper can be described in terms of the behavior of $h_{n}\left|\hat{g}^{*}(\xi, \eta)\right|$ along single lines through the origin in the $(\xi, \eta)$ plane. While local behavior is highly oscillatory, global behavior is generally monotone decreasing and convex on $\xi \geq 0$. This is shown in Fignre 4(A) for a typical SEM image along the line $\eta=0$. and sinnilar beliavior is found along other lines through the origin in the $(\xi . \eta)$ plane. A least squares fit to the oseillatory 
A

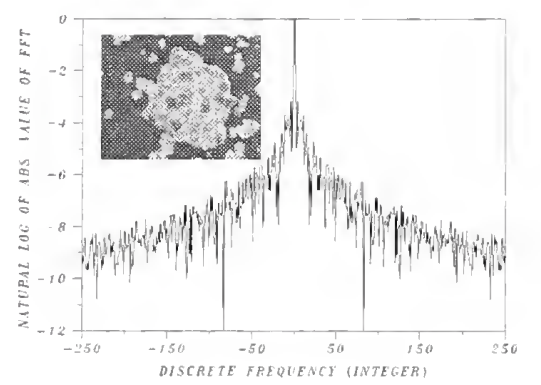

B

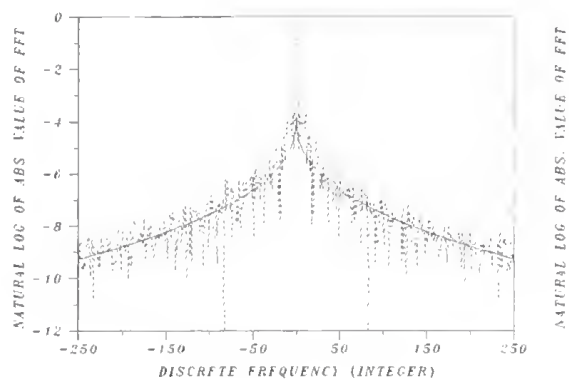

Figure 4: APEX detection of psf parameters for types of images considered in this paper. (A) Behavior of logarithm of normalized Fourier transform, ln $\left|\hat{g}^{*}(\xi, 0)\right|$, in typical SEM inage $g(x, y)$. White local behavior is highly oscillatory, global behavior is generally monotone decreasing and convex on $\xi \geq 0$. (B) Least squares fit to $\ln \left|\hat{g}^{*}(\xi, 0)\right|$ with $u(\xi)=-\alpha|\xi|^{2 \beta}-3.85$, (solid line). Fit develops well-formed cusp at $\xi=0$ and returns $\alpha=0.5346, \beta=0.2097$.

trace in Figure 4(A) with a smooth curve, provides a good representation of this global nonotone convexity property on $\xi \geq 0$. (A convex function is such that given any two distinct points $A$ and $B$ on its graph, the straight line segment joining $A$ and $B$ lies above the graph.) Many SEM images exhibit similar globally monotone convex behavior in Fourier space. Moreover, such behavior is also found in other types of inagery, unrelated to electron microscopy. In Ref. 3, a large class of images with that property was exhibited and denoted by W. The SEM inages considered here may be loosely characterized as being in class W. Not all blurred images may be so characterized. Application of the APEX method to cases where global behavior in $h\left|\hat{g}^{*}(\xi, \eta)\right|$, away from the origin, is nonotone decreasing and concave, are discussed in Ref 3. Use of the APEX nnethod in the manner described below is intended only for blurred inages whose Fourier space behavior is analogous to that shown in Figure $4(\mathrm{~A})$.

\section{Marching backwards in time and the APEX method}

The APEX method is based on the following observations. In the basic relation

$$
g(x, y)=\ln (x \cdot y) \otimes f_{e}(x, y)+n(x, y)
$$

we may safely assume that the noise $n(x, y)$ satisfies

$$
\int_{R^{2}}|n(x \cdot y)| d x d y \ll \int_{R^{2}} f_{e}(x \cdot y) d x d y=\sigma>0
$$

so that.

$$
\left|\hat{n}^{*}(\xi \cdot \eta)\right| \ll 1 .
$$

Consider the case where the off is a pure Lévy density $\hat{h}(\xi, \eta)=e^{-\alpha\left(\xi^{2}+\eta^{2}\right)^{\beta}}$. Since $g=g_{e}+n$.

$$
\ln \left|\hat{g}^{*}(\xi, \eta)\right|=\ln \left|e^{-\alpha\left(\xi^{2}+\eta^{2}\right)^{\beta}} \hat{f}_{e}^{*}(\xi, \eta)+\hat{n}^{*}(\xi, \eta)\right| \text {. }
$$



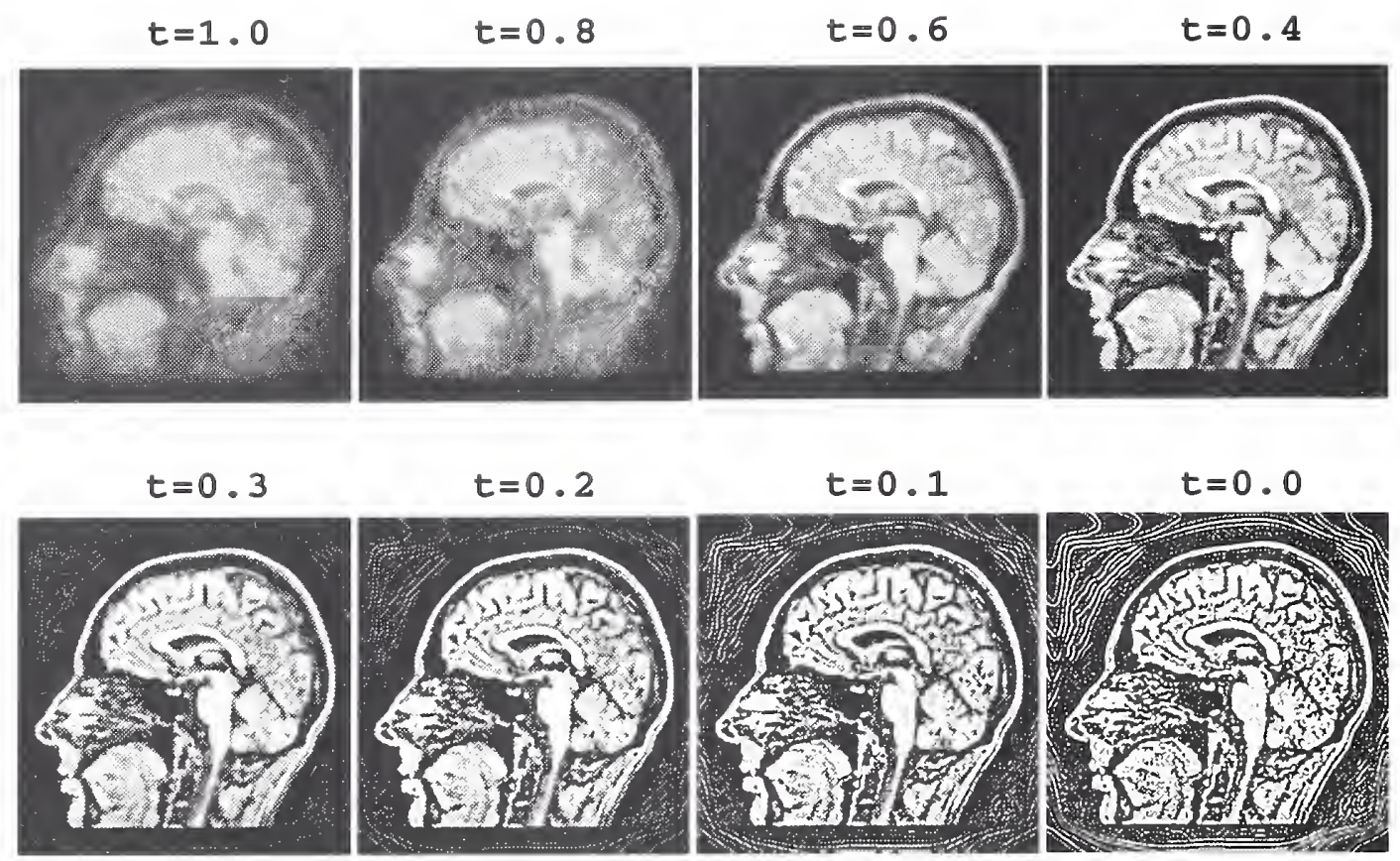

Figure 5: Enhancenent of 8-bit blurred MRI brain image by marching backwards from $t=1$ with APEX-detected psf. Sequence shows gradual increase in resolution as $t$ decreases. Undesirable artifacts near $t=0$ indicate progression backwards in time has continued too far. Here, best results orcur at values of $t$ such that $0.3 \leq t \leq 0.4$.

Let $\Omega=\left\{(\xi, \eta) \mid \xi^{2}+\eta^{2} \leq \omega^{2}\right\}$ be a noighborhood of the origin where

$$
e^{-\alpha\left(\xi^{2}+\eta^{2}\right)^{\beta}}\left|f_{e}^{*}(\xi, \eta)\right| \gg\left|\hat{n}^{*}(\xi, \eta)\right|
$$

Such an $\Omega$ exists since (19) is true for $\xi=\eta=0$ in view of (17). The radius $\omega>0$ of $\Omega$ decreases as $\alpha$ and $n$ increase. For $(\xi, \eta) \in \Omega$ we have

$$
\ln \left|\hat{g}^{*}(\xi, \eta)\right| \approx-\alpha\left(\xi^{2}+\eta^{2}\right)^{\beta}+\ln \left|\dot{f}_{e}^{*}(\xi, \eta)\right| .
$$

Because of the radial symmetry in the psf, it is sufficient to consicler (20) along a single line through the origin in the $(\xi, \eta)$ plane. Choosing the line $\eta=0$, we have

$$
\ln \left|\hat{g}^{*}(\xi, 0)\right| \approx-a|\xi|^{2 \beta}+\ln \left|\hat{f}_{e}^{*}(\xi, 0)\right|, \quad|\xi| \leq \omega
$$

Some type of a-priori information about $f_{e}(x, y)$ is necessary for blind deconvolution. In (21), knowledge of $h\left|\hat{f}_{e}^{*}(\xi, 0)\right|$ on $|\xi| \leq \omega$ would immediately yield $\alpha|\xi|^{2 \beta}$ on that interval. Moreover, any other line through the origin could have been used in (20). However, such detailed knowledge is unlikely in practice. The APEX method seeks to identify a useful psf from (21) without any prior knowledge about $\ln \left|\hat{f}_{e}^{*}(\xi, 0)\right|$. The method assumes instead that $f_{e}(x, y)$ is a recognizable object, and typically requires several interactive trials before locating a suitable psf. As previously noted. such trial SECB restorations are easily obtained. Here, prior information about $f_{e}(x, y)$ is disguised 
in the form of user recognition or rejection of the restored image, and that constraint is applied at the end of the reconstruction phase, rather than at the begimming of the detection phase.

In the absence of infornation about $\ln \left|\hat{f}_{e}^{*}(\xi, 0)\right|$, we replace it by a negative constant $-A$ in (21). For any $A>0$, the approximation

$$
\ln \left|\hat{g}^{*}(\xi, 0)\right| \approx-\alpha|\xi|^{2 \beta}-A
$$

is not valid near $\xi=0$, since the curve $u(\xi)=-\alpha|\xi|^{2 \beta}-A$, has $-A$ as its apex. Choosing a value of $A>0$, we best fit $\ln \left|\hat{g}^{*}(\xi, 0)\right|$ with $u(\xi)=-\alpha|\xi|^{2 \beta}-A$ on the interval $|\xi| \leq \omega$. using nonlinear least squares algorithms. The resulting fit is close only for $\xi$ away from the origin. The returned values for $\alpha$ and $\beta$ are then used in the SECB deblurring algorithm. Different values of $A$ return different pairs $(\alpha, \beta)$. Experience indicates that useful values of $A$ generally lie in the interval $2 \leq A \leq 6$. Increasing the value of $A$ decreases the curvature of $u(\xi)$ at $\xi=0$, resulting in a larger value of $\beta$ together with a smaller value of $\alpha$. A value of $A>0$ that returns $\beta>1$ is clearly too large, as $\beta>1$ is impossible for probability density functions. ${ }^{8}$ Decreasing $A$ has the opposite effect, producing lower vahues of $\beta$ and higher values of $\alpha$. As a rule, deconvolution is better behaver at lower vahues of $\beta$ than it is when $\beta \approx 1$. A significant discovery is that an image blurred with a pair $\left(\alpha_{0}, \beta_{0}\right)$ can often be successfully deblurred with an appropriate pair $(\alpha, \beta)$, where $\alpha>\alpha_{0}$ and $\beta<\beta_{0}$. An example of this phenomenon was shown in Fignre 2(D) in connection with the blurred New Orleans cathedral image. An effective interactive framework for performing the above least squares fitting is the fit command in DATAPLOT. ${ }^{21}$ This is a high-level English-syntax graphics and analysis software package developed at the National Institute of Standards and Technology. This software tool was used throughout this paper.

The following version of the APEX method, using the SECB marching backwards in time option (11). has been found useful in a variety of image enhancement problems where the image $g(x, y)$ is such that $\ln \left|\hat{g}^{*}(\xi, 0)\right|$ is generally globally monotone decreasing and convex, as shown in Figure $4(A)$. Choose a value of $A>0$ in (22) such that the least squares fit develops a well-formed cusp at $\xi=0$, as shown in Figure $4(\mathrm{~B})$. Using the returned pair $(\alpha, \beta)$ in the SECB methor, obtain a sequence $u^{\dagger}(x, y, t)$ of partial restorations (11), as $t$ decreases from $t=1$, as illustrated in the MRI image sequence in Figure 5. With a good choice of $A$, high quality restorations will be found at positive values of $t$, and these will gradually deteriorate as $t \rightarrow 0$. Typically, the restoration at $t=0$ will exhibit undesirable artifacts, indicating that continuation backwards in time has proceeded too far in (9). If the pair $(\alpha, \beta)$ produces a high quality restoration at $t=t_{1}>0$, the pair $\left(\alpha_{1}, \beta\right)$, where $\alpha_{1}=\left(1-t_{1}\right) a$, will produce the same quality results at $t=0$. In gencral, there will be nuany values of $A$ in $(22)$ returning pairs $(\alpha, \beta)$ that produce good reconstructions at sonle $t_{\alpha \beta}>0$. $\mathrm{A}$ large number of distinct pairs $\left(\alpha^{*}, \beta^{*}\right)$ can thus be found that produce useful, but distinct, results at $t=0$.

We have been assuming $h(\xi, \eta)$, to be a pure Lévy otf in (15). For more gencral class G otfs (2), we may still use the approximation $\ln \left|\hat{g}^{*}(\xi, 0)\right| \approx-\alpha|\xi|^{2 \beta}-A$, and apply the same technique to extract a suitable pair $(\alpha, \beta)$ from the blurred image. Here, the returned APEX vahues may be considered representative values for the $\alpha_{i}, \beta_{i}$ in (2), that produce a single pure Lévy otf aproximating the composite otf. 


\section{Application to SEM images}

It will be helpful to recall some basic properties of APEX processing in the discussion below. Given a sharp image $f(x, y)$, convolution of that image with any class $\mathrm{G}$ psf to form a blurred image $g(x, y)$, is mathematically equivalent to a heat conduction process in which bright areas in $f(x, y)$ correspond to hot spots, and dark areas to cold spots. As time progresses, heat conduction acts so as to diminish temperature differences. As a result, bright areas in $f(x, y)$ become dimmer in $g(x, y)$, while dark areas in $f(x, y)$ become lighter in $g(x, y)$. This causes a smoothing out of sharp edges, a loss of structural detail. and a decrease in contrast in $g(x, y)$.

APEX deblurring is the converse process. Given a blurred image $g(x, y)$, deconvolution of that image with a class $\mathrm{G}$ psf is equivalent to a reverse heat conduction process. Now, some light areas in $g(x, y)$ become brighter, while some gray areas become darker. There is a sharpening of edges, a gain in structural detail, and a necessary increase in contrast. Inevitably, there is also an increase in noise. By performing the deconvolution in slow motion, using the marching backwards in time option (11), we can monitor this reverse heat flow, and termimate the process at some time $t_{0}>0$ before brightness, contrast, or noise, become excessive.

Our first reconstruction experiments are displayed in Figures 6 through 10. In each of Figures 6 , 7 and 8, the top row contains the original SEM images that were used as input data into the APEX method. The bottom row contains the corresponding APEX-processed images. The middle row in each of these Figures was synthesized after acquiring and viewing tle bottom row images. To minimize the effects that contrast and brightness have on perception, the middle row images were created by readjusting contrast and brightmess in the original top row images, so as to more closely match that found in the bottom row as a result of APEX processing. Therefore, comparing the top row with the bottom row in Figures 6-8 shows the full effect of APEX processing, while comparing the middle row with the bottom row isolates the sharpening aspect of APEX processing. As might be expected, the vivid differences between these three rows, which are immediately apparent on a modern high-resolution computer screen, have become muted on the printed page. Accordingly, use of a magnifying glass may be helpful in parts of the following discussion. In Figures 9 and 10 , selected magnified portions of the contrast-enhanced and APEX-processed images in Figures 6-8 are compared. These enlargements provide good illustrations of the detection of fine structure as a result of APEX sliarpening.

All original micrographs were input as 8 -bit $512 \times 512$ images, although smaller sub images are displayed in some cases. Figures $6(\mathrm{~A}), 6(\mathrm{D})$ and $7(\mathrm{~A})$ are original images taken by John Small (NIST), on a Hitachi S-4500 field emission scanning electron microscope. All three images are micrographs of a complex multi-form crystalline compound of mercury. The field of view is $10 \mu \mathrm{m}$ in Figure 6(A), 200 $\mathrm{mm}$ in Figure 6(D), and $20 \mu \mathrm{m}$ in Figure $7(\mathrm{~A})$. Figure $7(\mathrm{D})$ is an image of a $2 \mu \mathrm{m}$ diameter fly ash particle on a Nuclepore filter. The filter was a backup for an impactor air sampler. The image was scamned from a Polaroid print taken by John Small (NIST) in the 1970's. on a Cambridge SEM ${ }^{\dagger}$ at the University of Maryland. Figure $8(\mathrm{~A})$ is a micrograph of a dust particle from an air vent. This is a complex agglomerate of biological and mineral particles. Figure 8(D) is

\footnotetext{
†Certain commercial equipment or products, including hardware and software components, are identified in this paper to adequately describe experimental procedures. Such identification does not imply recommendation or endorsement by the National Institute of Standards and Technology, nor does it mean that the equipment or products so identified are necessarily the best available for the purpose.
} 
A

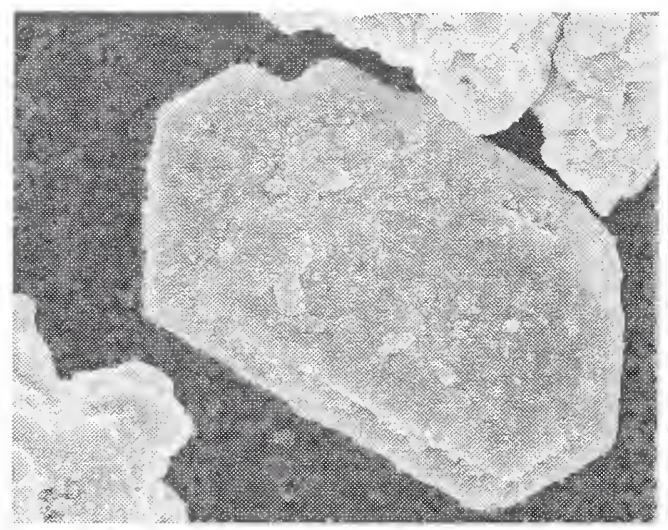

B
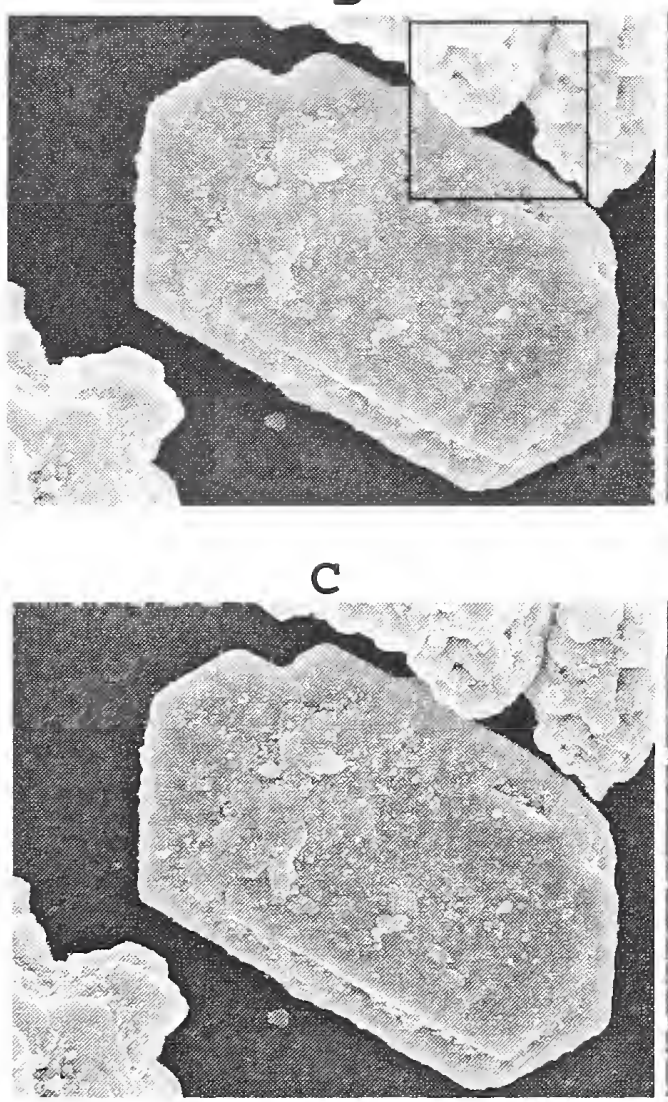

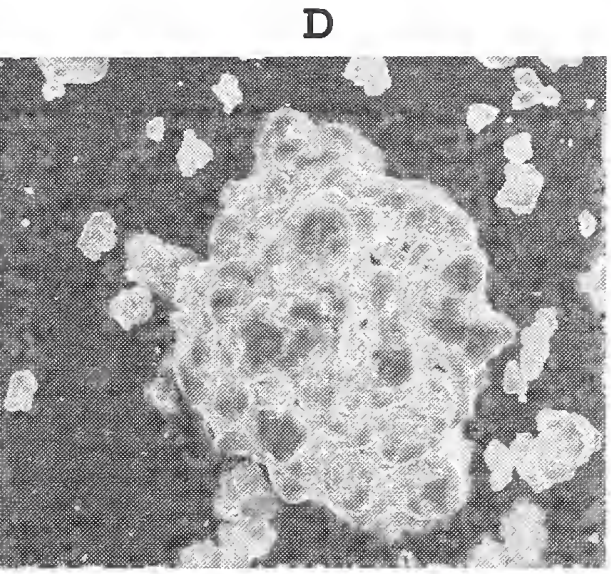

$\mathbf{E}$
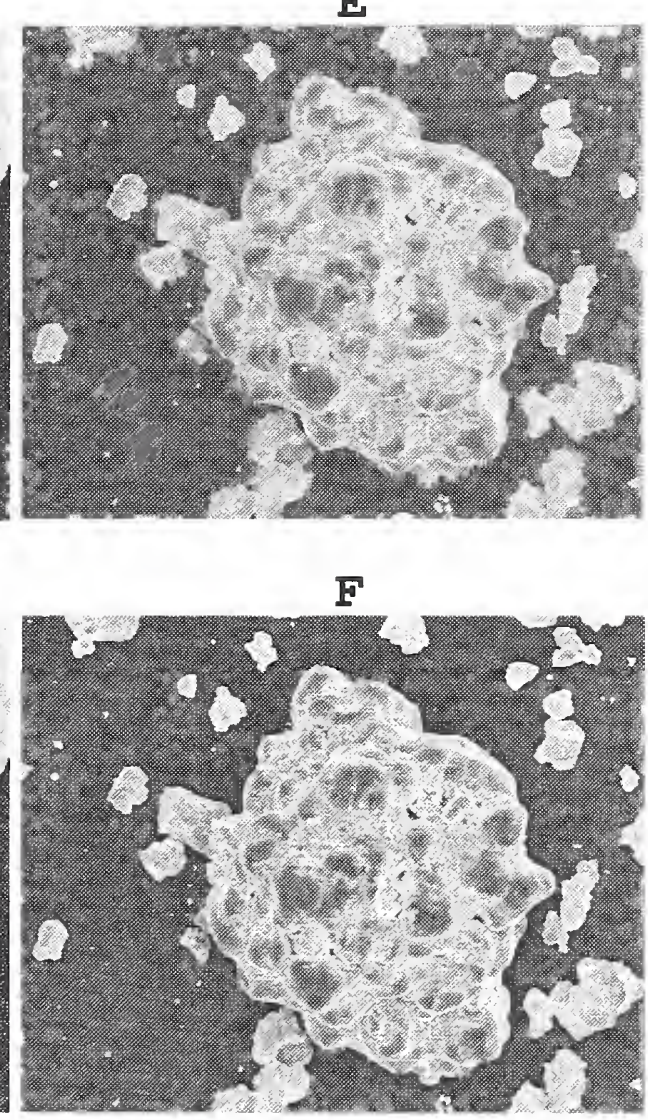

Figure 6: Top row images are original 8-bit $512 \times 512$ SEM micrographs, used as input data in APEX method. Field of view is $10 \mu \mathrm{m}$ in image (A) and $200 \mu \mathrm{m}$ in image (D). APEX-processed iniages are in bottom row. Middle row images obtained by readjusting contrast in top row images to match contrast in APEX-processed bottom row. Comparing bottom row with top row shows full effect of APEX processing. Comparing botton row with middle row isolates sharpening aspect of APEX metlod. Highlighted areas in middle row indicate regions of interest. See accompanving discussion in section 7 . 
A

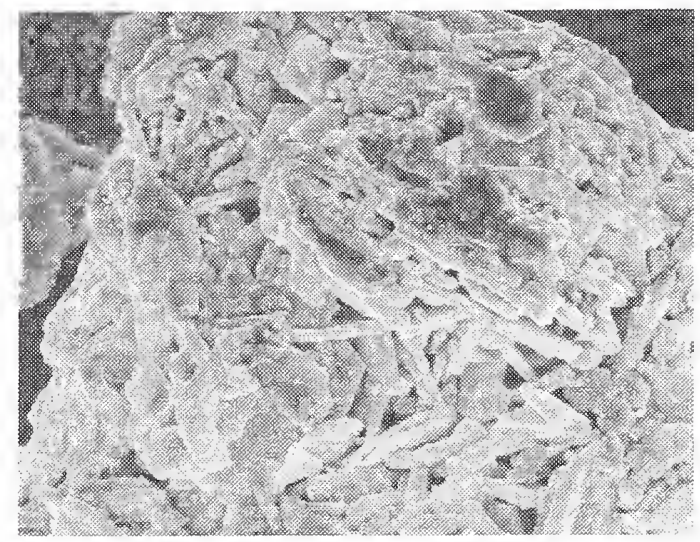

B
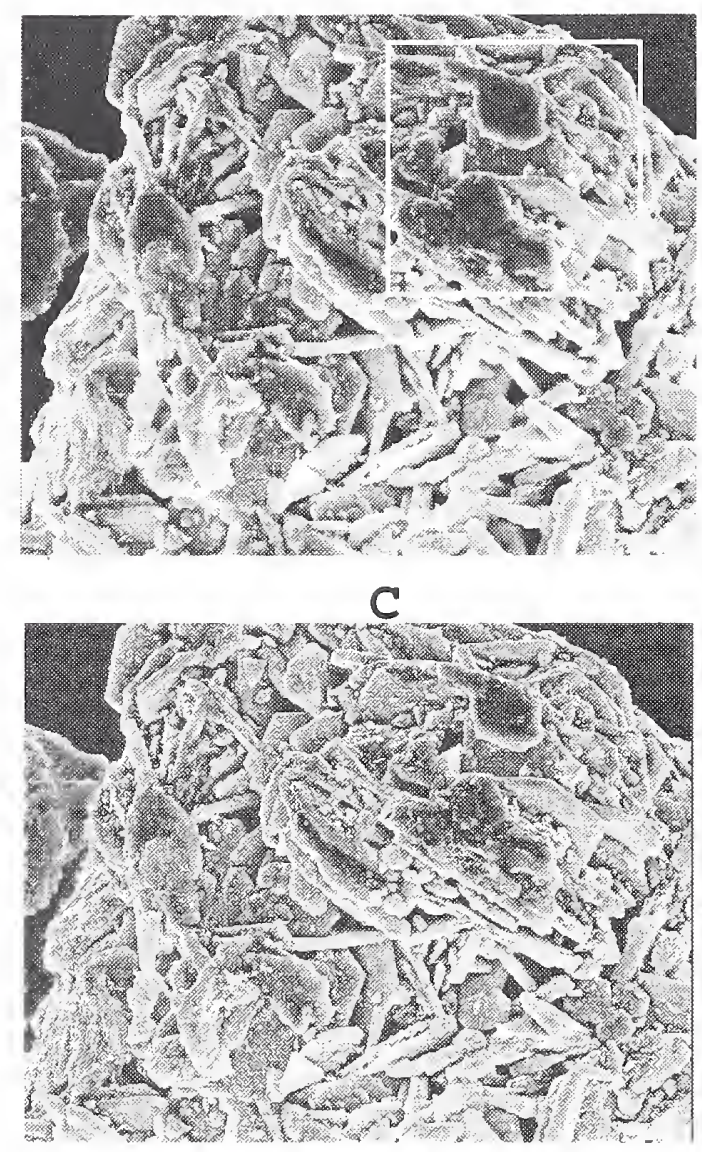

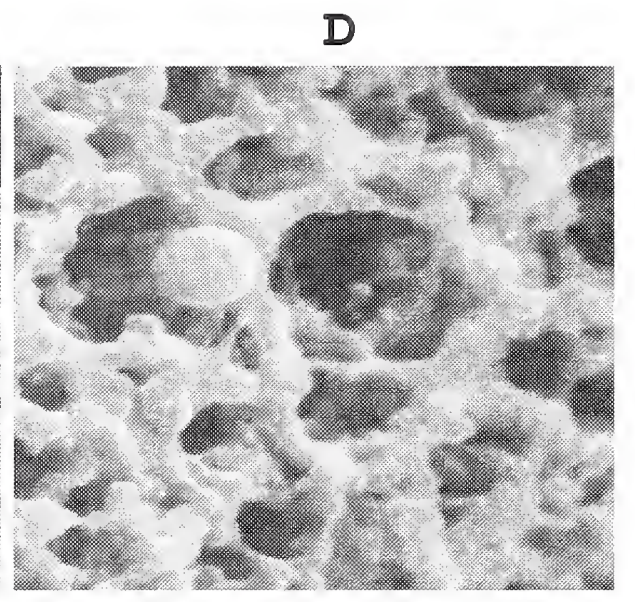

$\mathrm{E}$
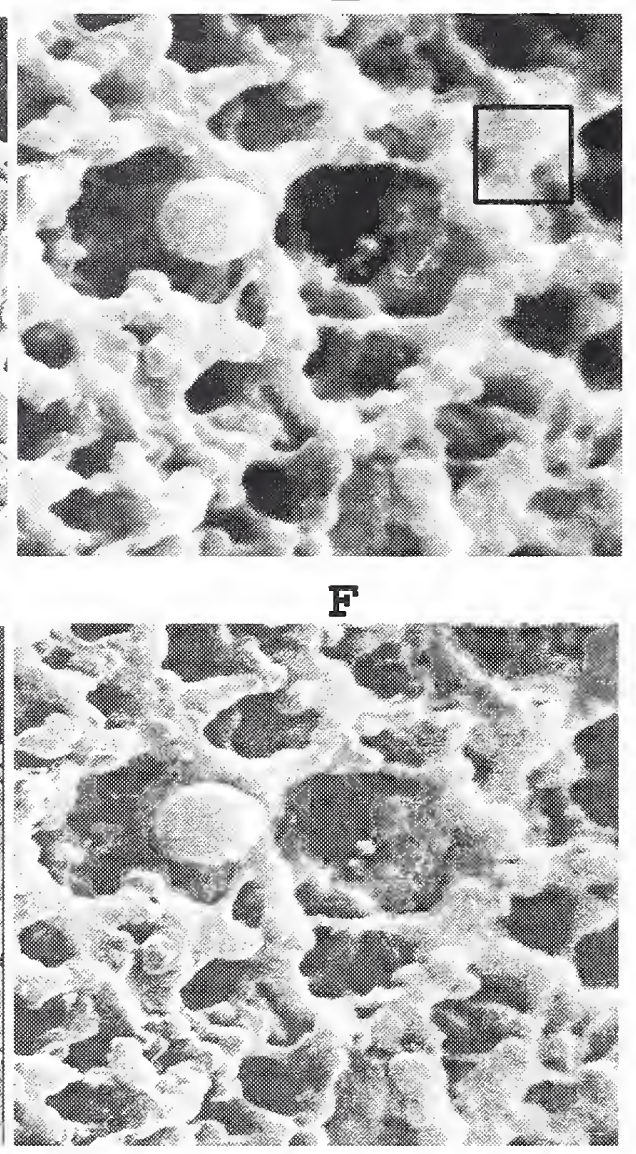

Figure 7: Top row images are original 8-bit $512 \times 512$ SEM micrographs, used as input data in APEX method. Field of view is $20 \mu \mathrm{m}$ in image (A) and $2 \mu \mathrm{m}$ in image (D). APEX-processed images are in bottom row. Middle row innages obtained by readjusting contrast in top row images to match contrast in APEX-processed bottom row. Comparing bottom row with top row shows full effect of APEX processing. Comparing bottom row with middle row isolates sharpening aspect of APEX method. Highlighted areas in middle row indicate regions of interest. See accompanying discussion in section 7 . 
A

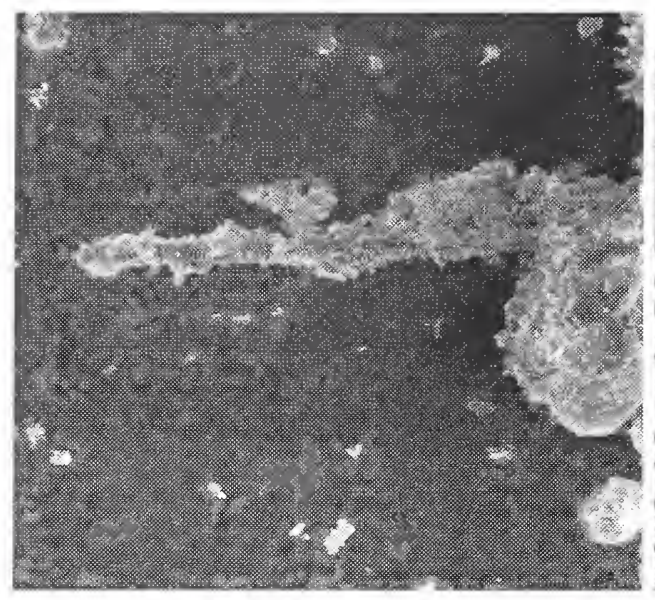

B

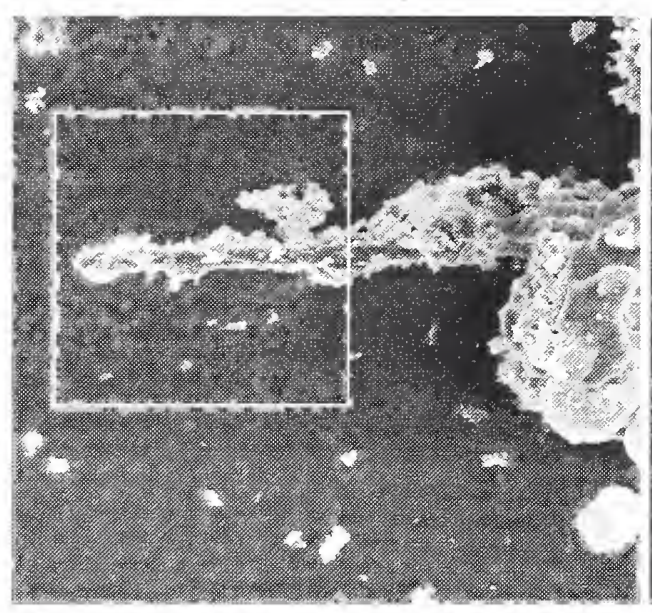

C

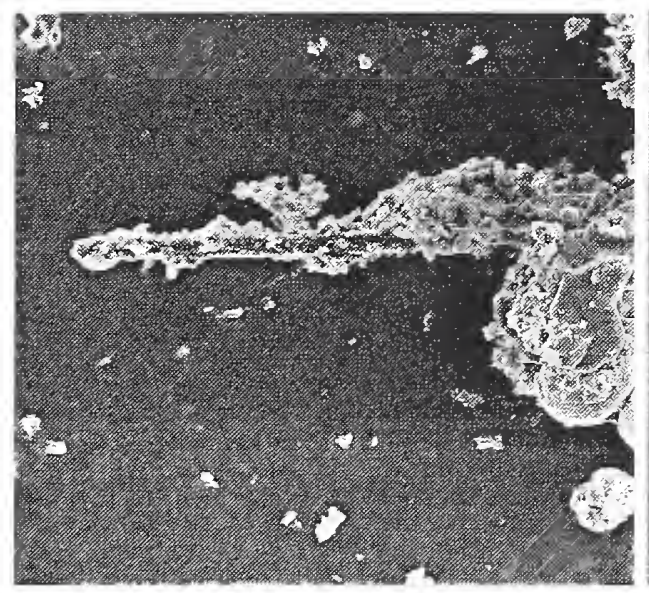

D

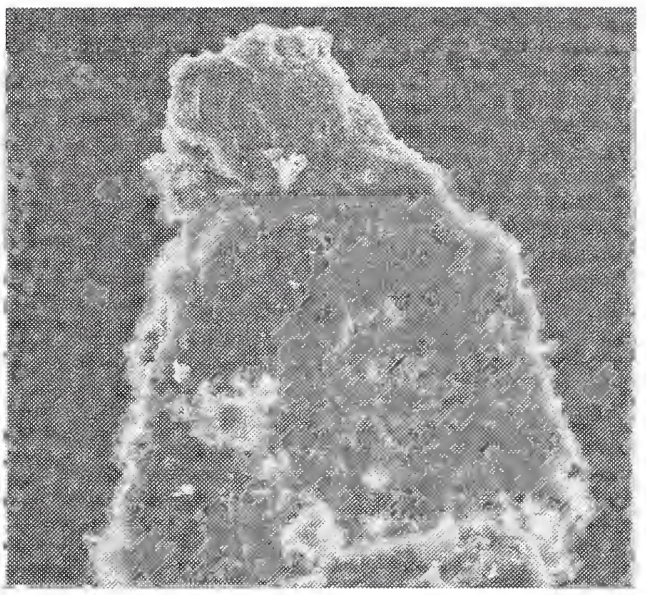

$\mathbf{E}$

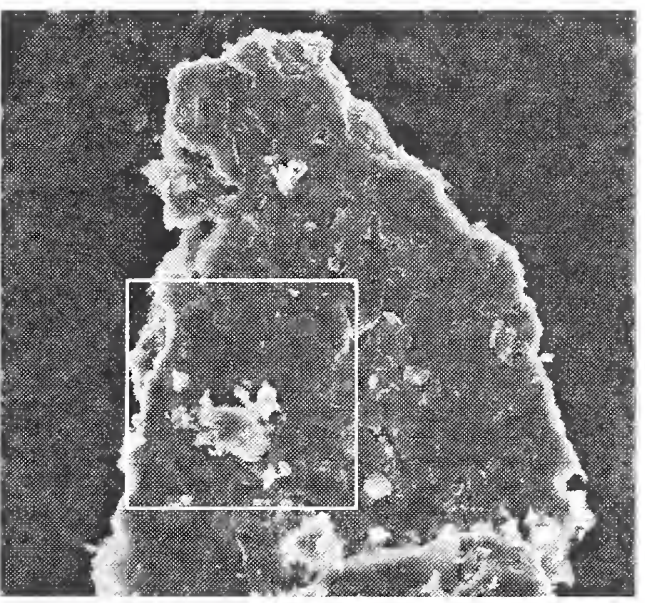

$\mathbf{F}$

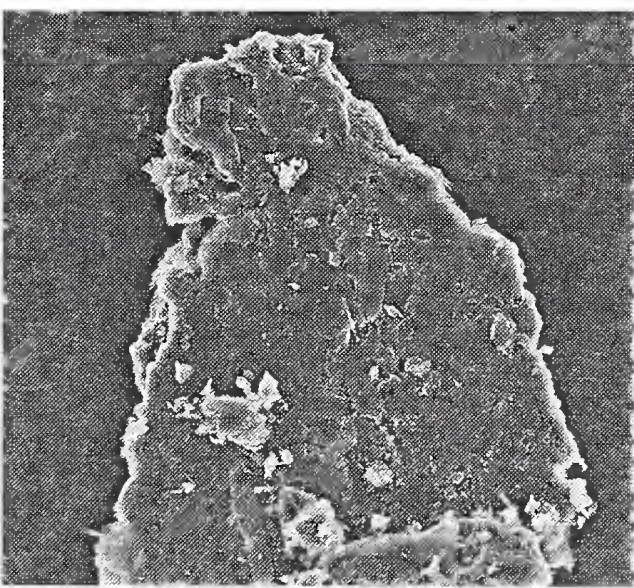

Figure 8: Top row images are $250 \mu \mathrm{m}$ field of view original 8-bit $512 \times 512$ SEM micrographs, used as input data in APEX method. APEX-processed inlages are in bottom row. Middle row images obtained by readjusting contrast in top row images to match contrast in APEX-processed botton row. Comparing bottom row with top row shows full effect of APEX processing. Comparing bottom row with middle row isolates sharpening aspect of APEX method. Highlighted areas in mithle row indicate regions of interest. See accompanying discussion in section $\bar{\tau}$. 
A

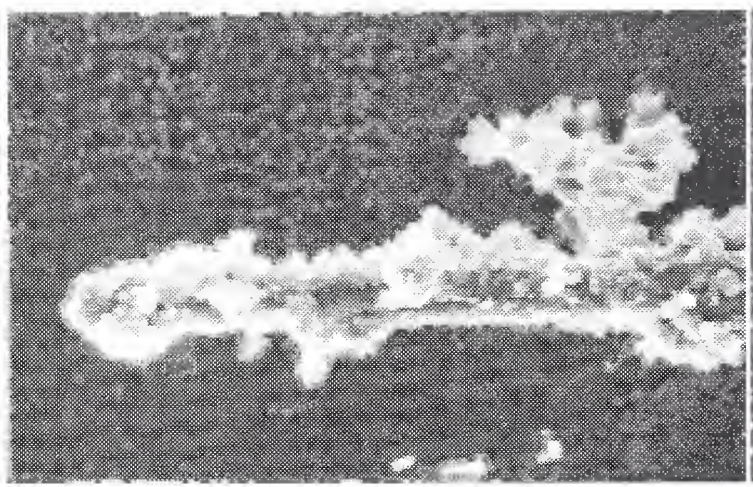

C

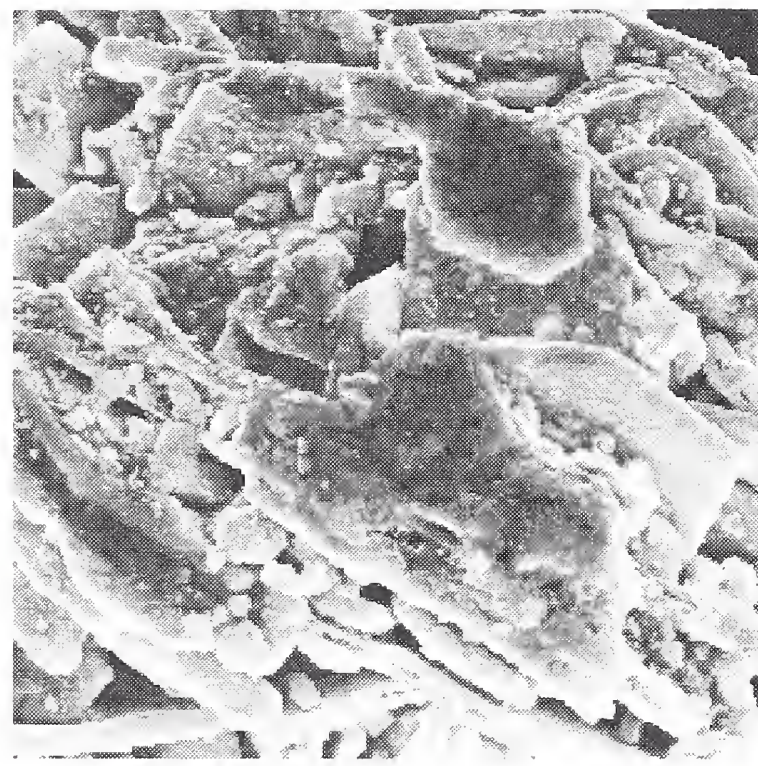

B

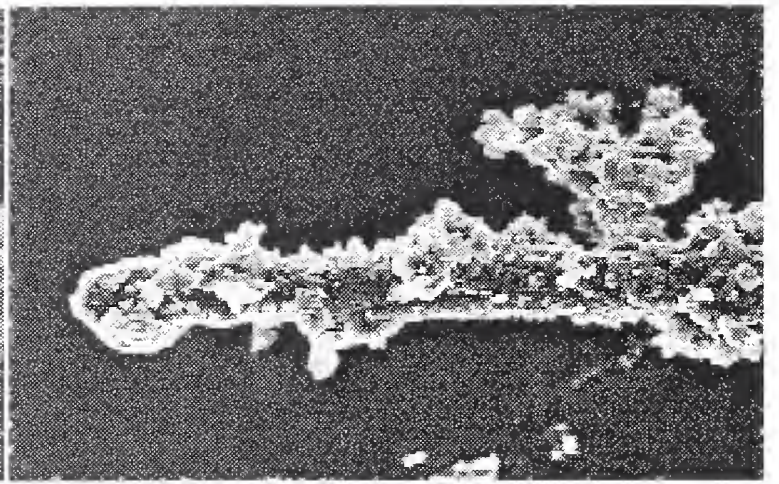

D
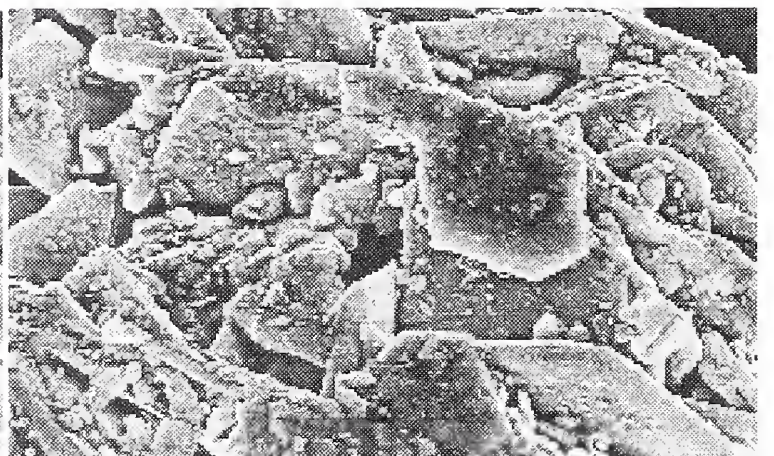

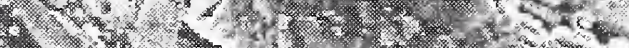
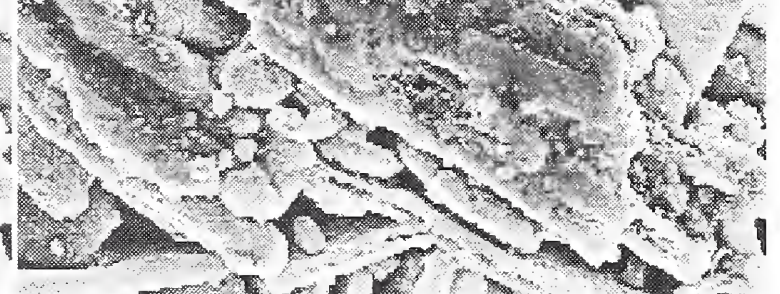

Figure 9: Comparison of contrast-enhanced and APEX-processed images illustrates detection of small-scale structure through image sharpening. (A) Magnified portion of image 8(B). (B) Corresponding portion of image $8(\mathrm{C})$. (C) Magnified portion of image $7(\mathrm{~B})$. (D) Corresponding portion of image $7(\mathrm{C})$. 
A

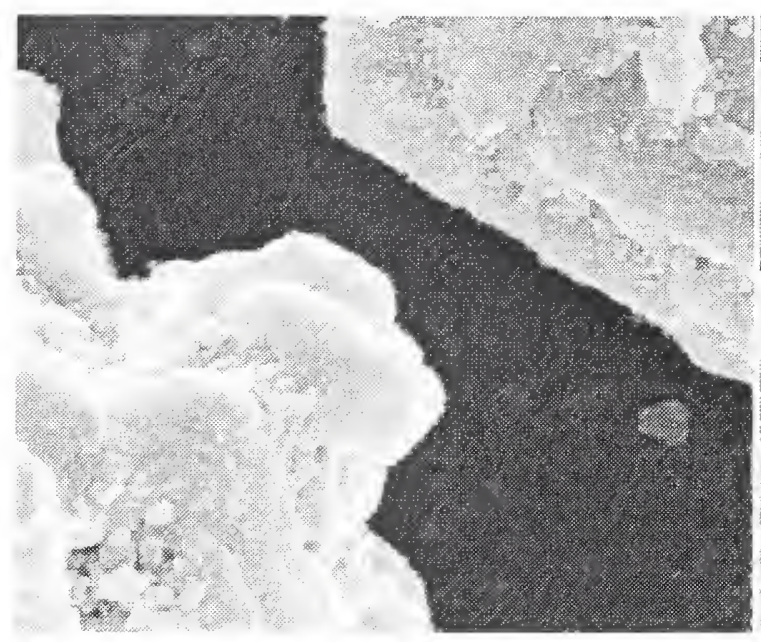

C

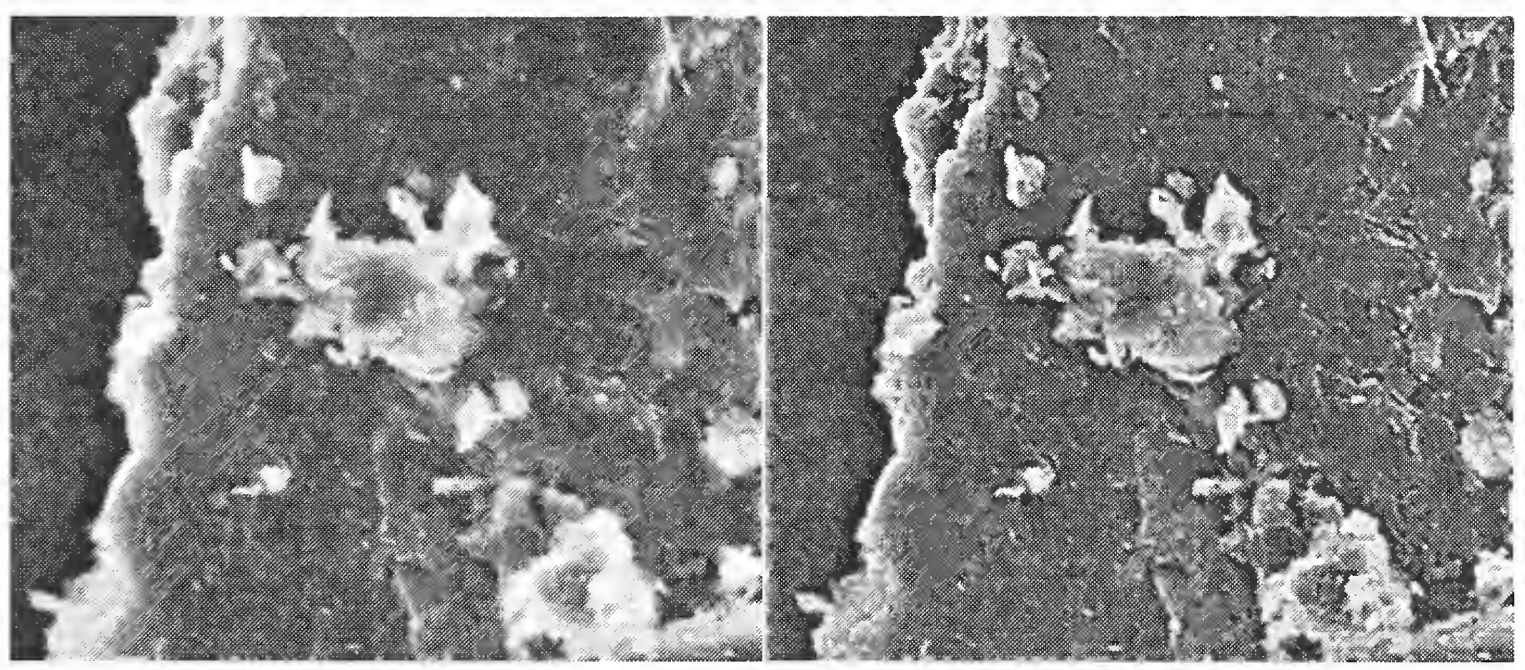

B

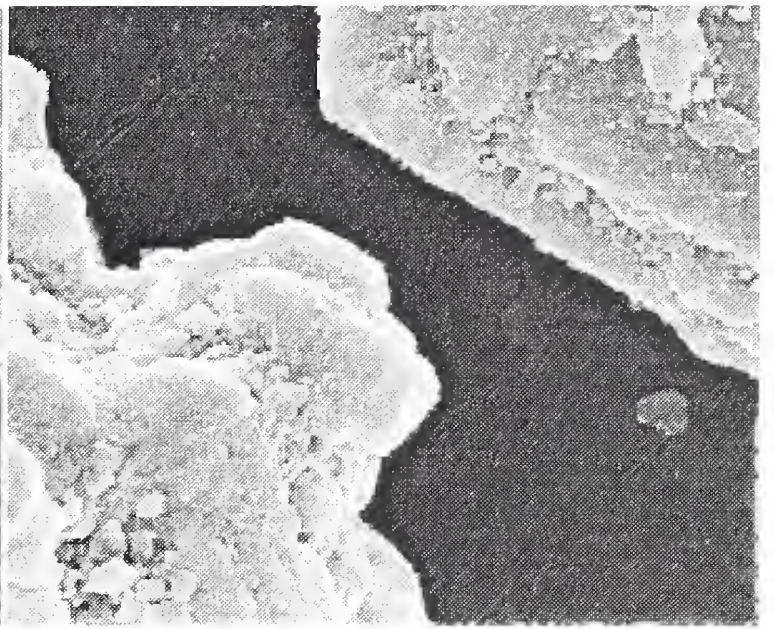

D 
a micrograph of a brass filing particle. Both of these were taken on a Hitachi S-4500, and the field of view is $250 \mu \mathrm{m}$ in each case.

In all cases in Figures 6-8, the APEX method was applied to the original top row images on the discrete frequency interval $|\xi| \leq 256$, and with an apex value of $A=3.85$. This produced a well-defined cusp at $\xi=0$ as illustrated in Figure $4(B)$. Different pairs $(\alpha, \beta)$ were detected for each image. The SECB method was then applied with the detected pairs $(\alpha, \beta)$, and with $s=0.001$ and $K=1.0$. Values of $t_{0}>0$ were chosen in each case to select the 'best' reconstruction. This choice of $t_{0}$ is partly subjective, but also depends in part on the particular features that need to be resolved. In general, images that are less sharpened seem more pleasing to the eye, while images that are more sharpened make surface detail and small decorations visible, albeit with noticeable background noise.

In Figure 6(A) the detected psf vahues were $\alpha=0.6165, \beta=0.1913$, and and a value $t_{0}=0.8$ was used to produce Figure 6(C). Although the original image 6(A) appears sharp with adequate contrast, more fine surface detail on the central particle becomes visible in the contrast-enhanced inage 6(B). However, in enhancing the surface detail on the central particle in image 6(B), other parts of the image suffer. For example, detail near the bright edges in the lumpy objects in the upper right, (see highlighted area in Figure 6(B)), as well as detail in the lumpy objects in the lower left, has been washed out. On the other hand, the APEX-processed image 6(C) shows even more fine detail in the central particle, while also showing more fine surface structure on the lumps in upper right and lower left corners.

In Figure 6(D) the detected psf values were $\alpha=0.5346, \beta=0.2097$, and a value $t_{0}=0.88$ was used to produce Figure 6(F). Since image 6(D) already has high contrast, there is not much difference between it and image 6(E), the contrast-enhanced version of 6(D). However, the APEX image $6(\mathrm{~F})$ has even more contrast, which helps bring out fine surface detail barely visible in the other two images. The APEX image 6(F) also has sharper and brighter edges, making the threedimensional form form of this complex particle easier to understand.

In Figure 7(A) the detected values were $\alpha=0.6915, \beta=0.1641$, and $t_{0}=0.64$ was used to produce Figure $7(\mathrm{C})$. This particular sample has very complex and varied morphology, in addition to surface dusting or decoration of fine particles ahmost everywhere. This becomes clearly evident only in the APEX image 7(C). Mere contrast enhancentent does not produce as much detail in the lighlighted area in image $7(\mathrm{~B})$, as is visible in the corresponding area in $7(\mathrm{C})$. Moreover, contrast enhancement in $7(\mathrm{~B})$ also tends to obscure texture in the brighter areas, such as in the lower left corner. However, the APEX image clearly shows the texture in the lower left corner as well as in other bright areas. It also retains the thrce-dimensionality of the particle by not eliminating shading, as is often the case with high-pass filtering.

In Figure $7(\mathrm{D})$ the detected psf values were $\alpha=0.9311, \beta=0.1441$, and a value $t_{0}=0.4$ was used to produce Figure $7(\mathrm{~F})$. This image is unlike the other images, in that it was scanned from an old Polaroid print rather than scanned digitally on the microscope. Imperfections on the Polaroid print are detected by APEX processing, along with enhancing the texture in the sample. Some of that texture may be due to the print rather than to the sample itself. Nevertheless, this example is a useful illustration of the APEX method's ability to detect fine structure. Presumably, actual imperfections or sniall defects in some other sample might have been detected equally well. While the scratches near the center are visible in all three images, the scratch near the top right corner (see highlighted area in image $7(\mathrm{E})$ ), is clearly discernible only in the APEX image $7(\mathrm{~F})$. Further, 
the edges in the APEX inage are sharper or less washed out than in the other two inlages. This makes the inage have more depth; the structure in the lower left fuadrant appears closer than does the rest of the inlage.

In Figure 8(A) the detected psf values were $\alpha=0.2981, \beta=0.2210$, and a value of $t_{0}=0.44$ was used to produce Figure 8(C). Contrast enhancenent in Fignre 8(B) makes the complex form of the sample easier to see, while bringing out fine particles. However, as in the previous cascs, such enhancement also obscures detail in the brighter areas, as in the highlighted area in Figure 8(B), for example. The APEX image 8(C) has brighter edges than the original 8(A), and sharper edges than the contrast enhanced 8(B). Moreover, fine detail becomes visible both in the medinm and bright areas of the image.

In Figure 8(D) the detected psf values were $\alpha=0.7634, \beta=0.182 \pi$, and a value $t_{0}=0.6$ was used to produced Figure $8(\mathrm{~F})$. The contrast enhanced Figure $8(\mathrm{E})$ is easier on the eyes, but does not have more visible detail than does the original Figure 8(D). The APEX image 8(F) has thinner or less washed out edges, making fine detail (which in this image is mostly in the edges) nunch easier to see. The highlighted area in Figure $8(\mathrm{E})$ is one example of a structure that is nore sharply defined in Figure $8(\mathrm{~F})$.

In Figures 9 and 10, selected enlarged portions of some of the contrast-enlanced and APEXprocessed images in Figures 6-8 are displayed side by side. Comparing these enlargements emphasizes some of the points made above, and provides a good illustration of the level of fine structure that may be revealed as a result of APEX sharpening.

\section{Etched grass image and quantitative APEX sharpness analysis}

In periodic performance testing of scamning electron microscopes, sharpness degradation in the micrograph of a suitable test object is often used as an indicator of the need for maintenance. The properties of an ideal test object for this purpose are discussed in Refs. 1 and 2. These properties include geonetric requirements. as well as the ability to vield reasonably noiseless images with good contrast at high magnification. A silicon wafer with an etching artifact called 'grass' was found to meet these criteria, and was used in Ref. 2. In the present paper, the sanne grass sample, together with 'SEM Monitor' software, ${ }^{2}$ provides a useful evaluation of the APEX method.

The images in the top row in Figure 11 are original 8-bit $512 \times 512$ images obtained from the Hitachi S-4700 field emission scanning electron microscope. The field of view is $200 \mathrm{~nm}$ for all intages. Image $(A)$ is as sharp as could be achieved with optimal settings of the focusing (objective) and stigma control ( $\mathrm{X}$ and $\mathrm{Y}$ ) lenses. Image (D) is out of focus. It was taken with an objective lens setting somewhat above that used for image (A), the sharpest image. Image (G) is astignatic and was taken with the I' stigma control set to a non-optinal value. Images (C), (F), and (I) in Figure 11, are the corresponding APEX-processed images. Each of these was selected from a sequence of increasingly sharper inages, as illustrated with the MRI image sequence in Figure 5. As in Figures 6. 7 and 8, the middle row innges in Figure 11 were created by readjusting the contrast in the top row images, so as to more closely match that found in the bottom row images as a result of APEX processing.

The following APEX parameter values were used in all thee cases, $A=3.85$ on $|\xi| \leq 256, s=$ $0.001, K^{r}=1.0$, and $t_{0}=0.9$. However, different pairs $(\alpha, \beta)$ were detected for each image. For the 
A

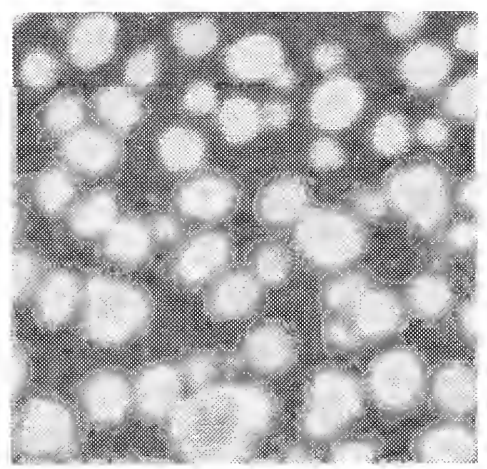

B

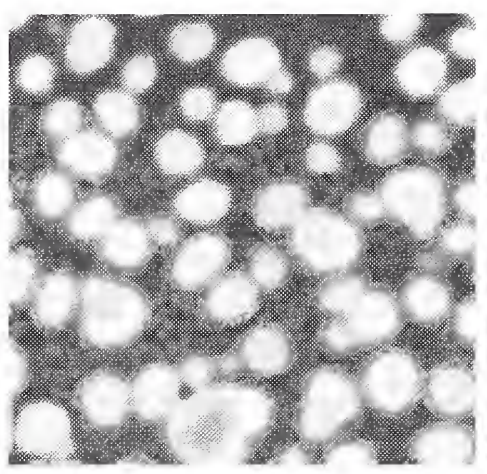

C

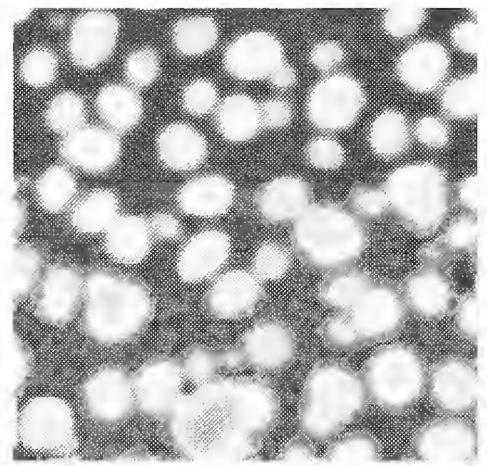

$\mathrm{D}$

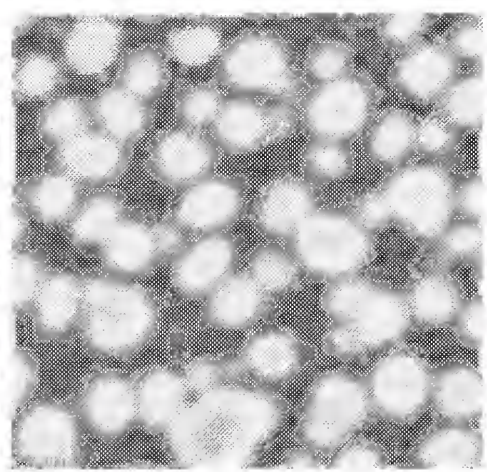

E

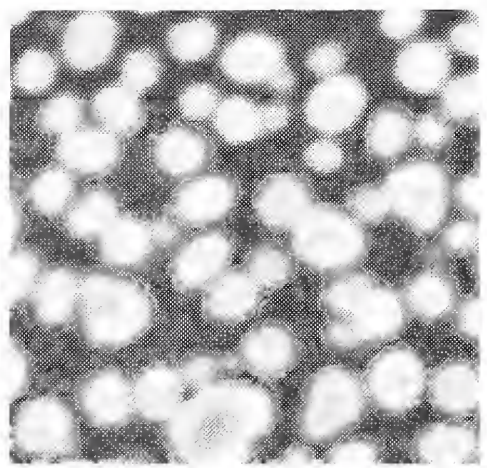

F

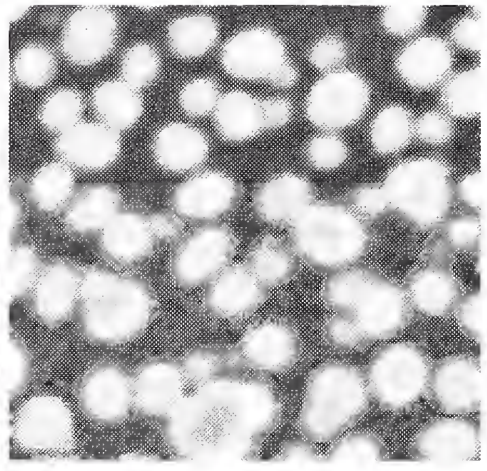

G

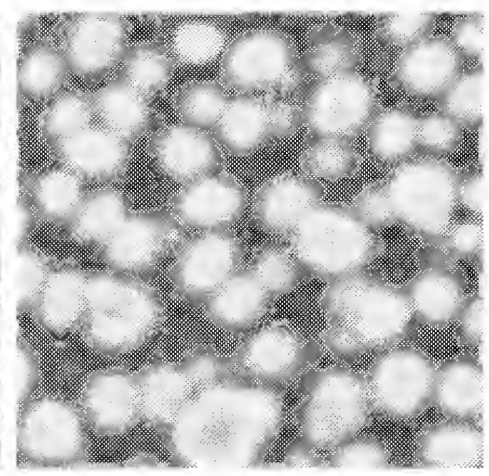

$\mathrm{H}$

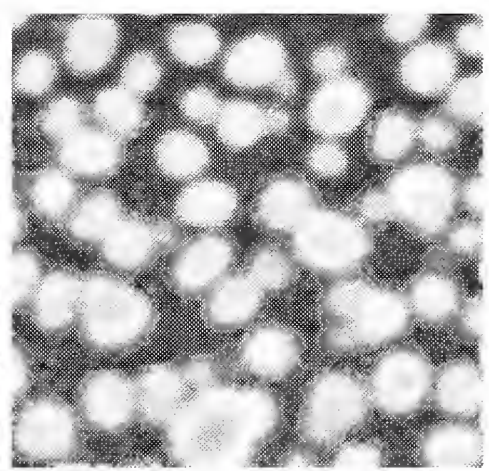

I

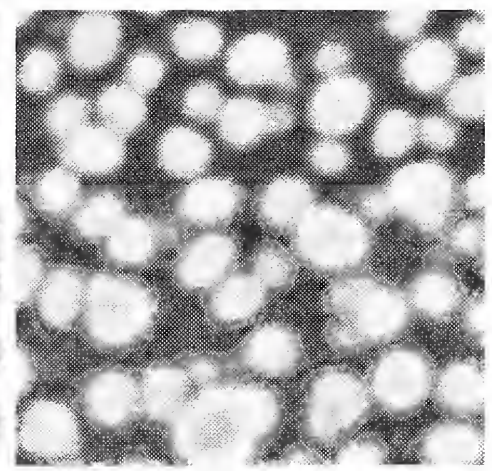

Figure 11: Top row images are $200 \mathrm{~nm}$ field of view original 8-bit $512 \times 512$ SEM nicrographs of 'grass' sample, used as input data in APEX procedure. Botton row images are corresponding APEX outputs. Image (A) is sharp, image (D) is out of focus, and inage (G) is astigmatic. Middle row intages obtained by adjusting contrast in top row inages to match contrast in APEX-processed bottom row. 'SEM Monitor' software indicates 15\% increase in sharpness after APEX processing. See Table 1 and accompanying discussion in section 8. 
sharp inage $(\mathrm{A}), \alpha=0.8508, \beta=0.1522$; for the out of focus image (D), $\alpha=1.1349, \beta=0.130 .5$; for the astignatic image $(\mathrm{G}), \alpha=1.1129, \beta=0.1321$. Again, comparing the top row with the bottom row in Figure 11 shows the full effect of APEX processing, while comparing the middle row with the bottom row isolates the sharpening aspect of the APEX method.

'SEM Monitor' is a hardware and software system designed to provide a quantitative framework for monitoring performance in scanning electron microscopes. Use of that system in connection with the above grass sample is discussed in Ref. 2. The system calculates several parancters, including a quantitative measure of image sharpmess. Here, we use that system to measure the effect of APEX processing on each of the three original micrographs in Figure 11. The results displayed in Table 1 indicate sharpness increases on the order of 15\% after APEX processing. Interestingly, this even holds true for the sharpest inage that could be achieved, image 11(A). This implies that APEX processing nay be used to extend an SEM's capability, by producing sharper imagery than is achievable under optimal settings.

\section{TABLE 1}

Sharpness improvement after APEX processing as measured by 'SEM Monitor'.

\begin{tabular}{|c|c|c|c|}
\hline Original sharpness & Detected pair $(\alpha, \beta)$ & APEX sharpness & Improvement \\
\hline Image $11(\mathrm{~A})=2.32$ & $\alpha=0.851, \beta=0.152$ & Inage $11(\mathrm{C})=2.68$ & $15.5 \%$ \\
\hline Image $11(\mathrm{D})=2.19$ & $\alpha=1.135, \beta=0.130$ & Image $11(\mathrm{~F})=2.51$ & $14.6 \%$ \\
\hline Image $11(\mathrm{G})=2.15$ & $\alpha=1.113, \beta=0.132$ & Inage $11(\mathrm{I})=2.45$ & $14.0 \%$ \\
\hline
\end{tabular}

\section{Concluding remarks}

This paper has demonstrated the use of a real-time blind deconvolution technique that can sharpen SEM micrographs. As shown in section 7 , such deconvolution enables detection of small-scale features not immediately apparent in the original micrograph. In section 8, APEX processing of ideal test sample micrographs produced measured increases in sharpness on the order of $15 \%$. While not all SEM images can be significantly improved, these results indicate the APEX methorl to be a useful tool in electron microscopy. Successful applications of APEX processing in several other imaging modalities, unrelated to SEM, have previously been documented. ${ }^{4}$

The APEX method is predicated on two assmmptions. The first assumption is that the blurred image $g(x, y)$ obeys the simple convolution equation (5) rather than a more general integral equation. The second assumption is that the point spread function $h(x, y)$ belongs to a restricted class of unimodal, radially synmetric, probability density functions, the class G defined in (2). It is not immediately obvious that the APEX method can be usefully applied in electron microscopy. 
The range of $\beta$ values that were detected and used in Figures 6-11, is interesting in its own right. The exponent $\beta$ expresses the degree of departure from more commonly occurring Gaussian densities where $\beta=1.0$. Here, $0.13 \leq \beta \leq 0.22$. A similar range of values values for $\beta$ was found in Ref. 4. As noted in Figure 2, there are several Lévy pairs $(\alpha, \beta)$ that can produce useful reconstructions, and higher values of $\beta$ might have been successfully employed. However, experiments indicate that the useful $\beta$ values in Figures $6-11$ typically lie in the range $0<\beta<1 / 2$. Future work will explore possible links between such $\beta$ values and physical processes mnderlying SEM imaging.

\section{Acknowledgements}

It is a pleasure to acknowledge the helpful advice provided by John Villarrubia, of the Precision Engineering Division at NIST, whose observations clarified some important points and led to significant improvements in the presentation.

\section{References}

[1] M. T. Postek and A. E. Vladár, "Innage sharpness measurement in scanning electron microscopy-Part I". SCANNING, 20 (1998), pp. 1-9.

[2] A. E. Vladár, M. T. Postek and M. P. Davidson, "Image sharpness measurement in scanning electron microscopy-Part II", SCANNING, 20 (1998), pp. 24-34.

[3] A. S. Carasso, "Direct blind deconvolution", SIAM J. Appl. Math., 61 (2001), pp. 1980-2007.

[4] A. S. Carasso, "The APEX method in image sharpening and the use of low exponent Léry stable laws", (May 2001). Submitted for publication. See also National Institute of Standards and Technology, NISTIR \# 6749. Available from National Technical Information Service. Springfield, VA 22161.

[5] A. S. Carasso, "Image restoration and diffusion processes", Proc. SPIE. 2035 (1993), pp. 255 266.

[6] A. S. Carasso, "Error bounds in nonsmooth image deblurring", SIAM J. Math. Anal., 28 (1997), pp. 656-668.

[7] A. S. Carasso, "Linear and nontinear image deblurring: a documented study", SIAM J. Numer. Anal., 36 (1999), pp. 1659-1689.

[8] WV. Feller, An Introduction to Probability Theory and its Applications, Vol. 2, Second ed., Wiley, New York, 1971.

[9] O. Barndorff-Nielsen, T. Mikosch and S. Resnick Eds., Lévy Processes-Theory and Applications, Birkliauser. Boston, 2001. 
[10] B. B. Mandelbrot, Fractals. Wr. H. Freeman, San Francisco, 1977.

[11] M. F. Shlesinger, G. M. Zaslarsky and U. Frisch Eds., Lévy Flights and Related Topres in. Physics, Lecture Notes in Physics \# 450, Springer-Verlag, New York, 199.5.

[12] W. A. Woyczyński. "Léry processes in the physical sciences", in Lévy Processes-Theory and Applications, O. Barndorff-Nielsen, T. Mikosch and S. Resnick Eds., Birkhauser, Boston 2001.

[13] C. B. Jolnson, "A method for characterizing electro-optical device modulation transfer functions", Photographic Science and Engineering, 14 (1970), pp. 413-415.

[14] C. B. Johnson, "Classification of electron-optical device modulation transfer function". Advances in Electronics and Electron Physics, Vol. 33B, (1972).

[15] C. B. Jolmson, "MTFs: a simplified approach". Electro-Optical Systems Design, 4, November 1972. pp. $22-26$.

[16] C. S. Williams and O. A. Becklund Introduction to the Optical Transfer Function, Wiley, New York, 1989.

[17] C. B. Jolnson, "Circular aperture diffraction limited MTF: approximate expressions", Applied Optics, 11 (1972), pp. 1875-1876.

[18] R. E. Hufnagel and N. R. Stanley, "Modulation transfer function associated with image transmission through turbulent media", J. Opt. Soc. Am., 54 (1964), pp. $52-61$.

[19] C. B. Johnson, "MTF parameters for all photographic films listed in Kodak pamphlet P-49", Applied Optics, 15 (1976), p. 1130.

[20] E. L. O'Neill, Introduction to Statistical Optics, Addison-Wesley, Reading, MA, 1963.

[21] A. Heckert and J. J. Filliben. DATAPLOT Reference Manual, available on World Wide Web at http://www.itl.nist.gov/div898/software/dataplot/document.litm

[22] IT. K. Pratt, Digital Inage Processing, Second ed., Wiley, New York, 1991.

[23] J. S. Villarrubia, A. E. Vladár, J. R. Lowney and M. T. Postek, "Edge determination for polycrystalline silicon lines on gate oxide", Proc. SPIE, 4344 (2001), pp. 14i-156.

[24] J. R. Lowney, "Application of Monte Carlo simulations to critical dimension metrology in a scanning electron microscope". Scanning Microscopy, 10 (1996), pp. 667-678. 


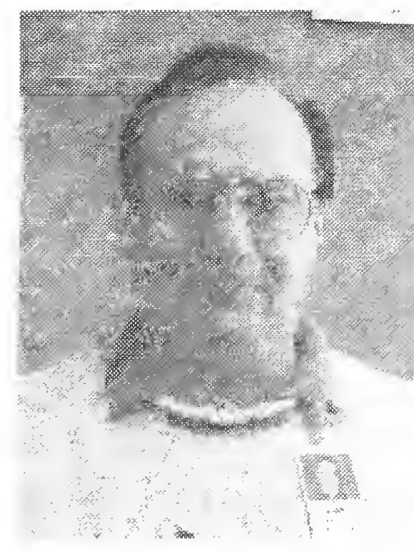

Alfred Carasso received the $\mathrm{Ph} . \mathrm{D}$. degree in Mathematics at the University of Wisconsin in 1968. He was a Professor of Mathematics at the University of New Mexico, and a Visiting staff Member at the Los Alamos National Laboratory, prior to joining the National Institute of standards and Technology (NIST) in 1982. His major research interests lie in the theoretical and computational analysis of ill-posed problems in partial differential and integral equations. together with their scientific applications. In 1976, he helped pioneer the use of time-reversed diffusion equations and 'slow motion' deconvolution in image deblurring, and has since developed novel inverse diffusion approaches in other types of system identification problems in science and engineering. He has done basic theoretical work in ill-posed inverse problems, and is a patentee in the field of image processing. He has authored numerous papers in the SIAM journals. and is an active speaker at applied mathematics colloguia, conferences, and national meetings.

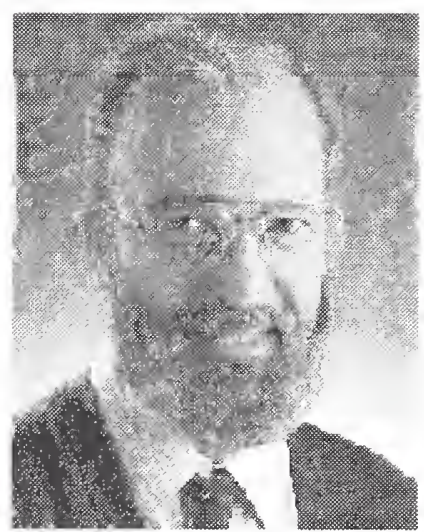

David Bright received the Ph.D. degree in Biophysics at Colorado State University in 1975. He has been a research chemist at the National Institute of Standards and Technology (NIST) since 1976, and has had an interest in computerassisted analysis since that time. In 1984, he joined the Microanalysis Group at the NIST Center for Analytical Chemistry to develop an image analysis system, IISPIX, and apply image analysis techniques to various research problems in that group. Scientists can use LISPIX to automate electron microscopes, identify arrays in electron diffraction patterns, enhance and correlate $\mathrm{X}$-ray maps, find and measure areas of interest in images, archive images, and exchange data and images with other image analysis programs. Bright uses LISPIX to develop new algorithms for various image processing applications. He has authored over 70 papers in image analysis since 1983, including an Interface article in Analytical Chemistry '91, and has given over 50 invited talks on the subject.

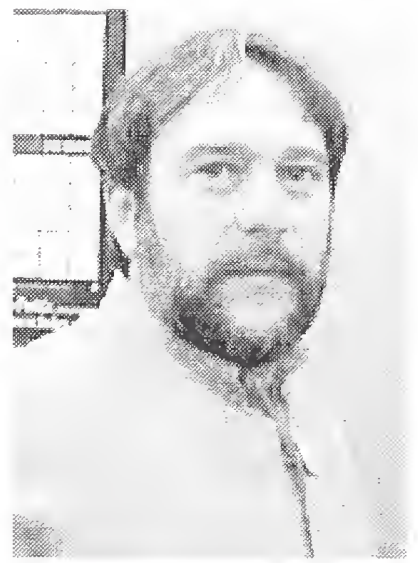

Andras Vladar received the $\mathrm{Ph} . \mathrm{D}$. degree in Electrical Engineering at the Technical University of Budapest, (Hungary), in 1984. He was a research scientist at the Hungarian Academy of Sciences, prior to his arrival in the USA in 1999. He has been a guest scientist at the National Institute of Standards and Technology (NIST), and a staff member at the Hewlett-Packard ULSI Research Laboratory, where he worked on the development of integrated circuit technologies. He is currently the scanning Electron Microscopy Project Leader in the Nanometer-Scale Metrology Group at NIST. He has helped define and implement basic standards for the measurement and monitoring of electron microscope imaging performance. His main research interests lie in Dimensional Metrology and Digital Imaging techniques related to scanning electron microscopy. 

